\title{
On the Dynamics of Tropical Cyclone and Trough Interactions
}

\author{
William A. Komaromi AND James D. DoYle \\ Naval Research Laboratory, Monterey, California
}

(Manuscript received 14 September 2017, in final form 15 May 2018)

\begin{abstract}
The interaction between a tropical cyclone (TC) and an upper-level trough is simulated in an idealized framework using Coupled Ocean-Atmosphere Mesoscale Prediction System (COAMPS) for Tropical Cyclones (COAMPS-TC) on a $\beta$ plane. We explore the effect of the trough on the environment, structure, and intensity of the TC. In a simulation that does not have a trough, environmental inertial stability is dominated by Coriolis, and outflow remains preferentially directed equatorward throughout the simulation. In the presence of a trough, negative storm-relative tangential wind in the base of the trough reduces the inertial stability such that the outflow shifts from equatorward to poleward. This interaction results in a $\sim 24-\mathrm{h}$ period of enhanced upper-level divergence coincident with intensification of the TC. Sensitivity tests reveal that if the TC is too far from the trough, favorable interaction does not occur. If the TC is too close to the trough, the storm weakens because of enhanced vertical wind shear. Only when the relative distance between the TC and the trough is 0.2-0.3 times the wavelength of the trough in $x$ and 0.8-1.2 times the amplitude of the trough in $y$ does favorable interaction and TC intensification occur. However, stochastic effects make it difficult to isolate the intensity change associated directly with the trough interaction. Outflow is found to be predominantly ageostrophic at small radii and deflects to the right (in the Northern Hemisphere) since it is unbalanced. The outflow becomes predominantly geostrophic at larger radii but not before a rightward deflection has already occurred. This finding sheds light on why the outflow accelerates toward but generally never reaches the region of lowest inertial stability.
\end{abstract}

\section{Introduction}

The overall role of the tropical cyclone (TC) outflow layer and its effect (if any) on the underlying storm intensity and structure is not fully understood. One traditional view, referred to as passive outflow, is that the strength and structure of the outflow is forced by or a slave to the strength of the primary vortex and associated low-level inflow layer without any meaningful feedback to storm intensity or structure (e.g., Van Sang et al. 2008). An alternative hypothesis, referred to as active outflow, is that a source of upper-level forcing that acts to accelerate or otherwise enhance the TC outflow can ultimately drive changes in the strength or structure of the vortex below (e.g., Sadler 1976; Holland and Merrill 1984; Nong and Emanuel 2003). Here, we do not seek to determine whether outflow is more likely to be passive or active. Instead, we explore the hypothesis that active outflow may contribute to

Corresponding author: William A. Komaromi, will.komaromi@ nrlmry.navy.mil
TC intensification under the right set of conditions, namely, during the interaction between a TC and an upper-level trough.

Several explanations exist in the literature as to why trough interaction would affect either the TC intensity or vortex structure. A number of studies argue trough interaction can enhance upper-level divergence, creating a more favorable environment for sustained convection (Sadler 1976; Merrill 1988a,b; Bosart et al. 2000). Other studies have argued that enhanced ascent associated with the trough's secondary circulation and/or the right-entrance region of a jet streak act to strengthen the secondary circulation associated with the vortex itself (Rodgers et al. 1991; Shi et al. 1997; Hanley et al. 2001; Merrill 1988a,b; Fischer et al. 2017). Sadler (1976) suggested that favorable TC interactions with upper-level troughs act to enhance upper-level divergence and establish a connection between the TC outflow and large-scale westerlies, ventilating and thereby strengthening the cyclone. Similarly, Holland and Merrill (1984) suggest that synoptically enhanced outflow may lead to an intensification of the TC because of enhanced 
convection and a contraction of the core. They hypothesize that differential angular momentum transports between this enhanced outflow and a shallow inflow layer immediately above and below the outflow layer in conjunction with the resultant enhanced stratospheric inflow and subsidence act to strengthen the warm core, providing further intensification. Additionally, Nong and Emanuel (2003) suggest a mechanism by which a moist upper-level potential vorticity (PV) anomaly can project downward along angular momentum surfaces to influence the surface vortex.

Another mechanism by which a trough may modify the TC environment is by modifying inertial stability $I$, defined as

$$
I^{2}=\left(f+\frac{2 v_{t}}{r}\right)(f+\zeta)
$$

where relative vorticity $\zeta=(1 / r)\left[\partial\left(r v_{t}\right) / \partial r\right], f$ is the Coriolis term, and $v_{t}$ is the tangential component of wind (Alaka 1961). TCs primarily interact with environmental flow at upper levels where $I$ is weaker, as $I$ is a measure of the resistance to motion in the radial direction (Schubert and Hack 1982; Shapiro and Willoughby 1982; Hack and Schubert 1986). Therefore, a reduction in I may allow stronger radial outflow to develop in the direction of low I (Holland and Merrill 1984; Rappin et al. 2011; Molinari and Vollaro 2014; Barrett et al. 2016). Molinari and Vollaro (2014) find positive radial wind $v_{r}$ to be maximized at the outer edge of the inertially unstable $(I<0)$ region and hypothesize that the length of time that the inertially unstable region persists may be related to the nature or length of the period of TC intensification. Komaromi and Doyle (2017) find the greatest radial acceleration of the radial wind to occur above $200 \mathrm{hPa}$ in a region of low $I$ less than $1 \times 10^{-4} \mathrm{~s}^{-1}$. Rappin et al. (2011) argue that a reduction in inertial stability north of the TC associated with the insertion of a westerly zonal jet minimizes the energy expenditure of forced subsidence by enhancing the outflow along a single narrow path, allowing radiational cooling to lessen the work of subsidence and thereby intensifying the TC. Generally, a trough north or west of a TC will reduce $I$ by reducing both $\zeta$ and $v_{t}$, thereby creating an environment more favorable for the development of outflow. While the direction of positive $v_{r}$ is often broadly consistent with the direction of lower $I$, Barrett et al. (2016) find the strongest outflow to occur $45^{\circ}-90^{\circ}$ to the right of the minimum $I$. They attribute this phenomenon to the fact that the outflow is modifying its own environment by reducing (enhancing) the $v_{t}$ term in the equation for $I$ counterclockwise (clockwise) of the region of strongest outflow. In this sense, the outflow is continuously "chasing" the region of lowest $I$ but can never quite reach it.

A number of studies have identified the flux convergence of angular momentum by the azimuthal eddies, or "eddy flux convergence" (EFC), as an additional mechanism by which environmental angular momentum associated with a trough can contribute to TC structural or intensity changes. EFC is defined as

$$
\mathrm{EFC}=-\frac{1}{r^{2}} \frac{\partial}{\partial r} r^{2} \overline{v_{r}^{\prime} v_{t}^{\prime}}
$$

where $v_{r}^{\prime}$ is the perturbation radial wind, $v_{t}^{\prime}$ is the perturbation tangential wind, and the overbar denotes the azimuthal mean, computed in storm-relative coordinates (Molinari and Vollaro 1990). Previous studies have suggested that the enhanced EFC may trigger structural changes that ultimately lead to intensification of TC (Molinari and Vollaro 1989, 1990; Molinari et al. 1995). Molinari and Vollaro $(1989,1990)$ propose that positive EFC triggers an inward-propagating maximum in $v_{r}$, which produces a balanced vortex response and thereby is associated with an inward-propagating region of vertical velocity $w$. This inward-propagating region of positive $w$ would then trigger a secondary eyewall formation, associated with a temporary weakening of the TC but followed by a stronger final intensity due to the convergence of angular momentum associated with the eddies. Leroux et al. (2013) found a similar evolution, with trough interaction triggering secondary eyewall formation for TC Dora in the Indian Ocean. In a follow-on study, Leroux et al. (2016) found an optimal position of the TC relative to the trough in order for favorable interaction to occur. However, for many real cases, the temporal window in which EFC promotes TC intensification will be short: positive EFC may occur too far from the TC if the trough is not nearby, while the detrimental effects of shear may be too great once the EFC occurs close enough to the storm to promote intensification (DeMaria et al. 1993; Titley and Elsberry 2000; Yu and Kwon 2005; Peirano et al. 2016).

While it has been argued that an increase in divergence, an increase in angular momentum via eddy flux convergence, and a reduction in inertial stability at upper levels associated with an approaching trough can enhance outflow and potentially lead to an intensification of the TC, troughs may also weaken the cyclone because of an attendant increase in vertical wind shear (Lewis and Jorgensen 1978). This concept of "good trough versus bad trough" was quantified over a large sample of cases by DeMaria et al. (1993) and later expanded upon and refined by Hanley et al. (2001) and Peirano et al. (2016). DeMaria et al. (1993) found TC 
intensification associated with enhanced EFC at upper levels associated with trough interaction in approximately one-third of their sample. Hanley et al. (2001) found favorable TC-trough interaction (intensifying the TC) to be slightly more common than unfavorable (weakening the TC) interaction. However, including more seasons and controlling for sample bias, Peirano et al. (2016) found troughs to be, on average, unfavorable for intensification. Hanley et al. (2001) also demonstrated composite differences in upper-level PV between intensifying and nonintensifying cases to be fairly minor, suggesting small changes in how the TC interacts with the trough and/or fairly subtle changes in shear can make a large difference in outcome. Kimball and Evans (2002) find the nature of the TC-trough interaction to be highly sensitive to the strength and depth of the trough, with results varying based upon initial conditions from a weak, asymmetric TC in one simulation to a symmetric $\mathrm{TC}$ that undergoes rapid intensification in another. They also note that while the trough modifies the structure of the TC, the TC also modifies the structure of the trough during the interaction.

There is also some overlap of dynamical processes involved in the interaction of a mature, purely tropical cyclone with an upper-level trough, which we have been discussing, and the tropical transition (TT) problem, in which the development of an initially weak low-level vortex into a TC is aided by the interaction with an upper-level trough. Cold upper-level temperatures associated with the trough steepen the lapse rates, which in an environment associated with cyclonic vorticity advection and forcing for ascent triggers deep convection over warm SSTs (Guishard et al. 2009; Bentley et al. 2016). Midlevel latent heat release associated with this convection can then act to erode the upper-level PV anomaly associated with the trough, allowing the low-level vortex to occlude, axisymmetrize in an environment of reduced shear, and develop tropical characteristics (Palmén and Newton 1969; Raymond 1992; Posselt and Martin 2004; Davis and Bosart 2003, 2004). However, Galarneau et al. (2015) found the difference in the strength and placement of the upper-level trough relative to the TC to be quite subtle, with the strength of the upper-level anticyclone north of the TC being a stronger differentiator between genesis and nongenesis. Bentley et al. (2017) also found differences between TT events in the presence of meridional troughs versus zonal troughs and cutoff lows, with stronger quasigeostrophic forcing likely required to initiate the necessary deep convection for TC formation in the presence of meridional troughs.

A number of studies have examined the interaction of a TC and a trough for real cases using reanalyses, model analyses, or real-data simulations (e.g., Riehl 1954; Sadler 1976, 1978; Holland and Merrill 1984; Molinari and Vollaro 1989, 1990; Hanley et al. 2001; Yu and Kwon 2005; Barrett et al. 2016; Rios-Berrios et al. 2016). Comparatively few studies exist in the literature that examine TC-trough interaction in a more fundamental manner such as in a modeling framework (Shi et al. 1990; Kimball and Evans 2002; Ge et al. 2008; Rappin et al. 2011). The idealized framework, which will be utilized in this study, allows one to control the number of degrees of freedom to disentangle and isolate the key dynamics. In this study, the dynamics of TC and trough interaction, including the effects of trough interaction on TC intensity change, will be explored. We also seek to systematically explore the effect of modifying the environmental inertial stability and the resulting effect on the strength, structure, and direction of the storm outflow. Last, predictability issues associated with TC intensity change and the relative positions of the $\mathrm{TC}$ and the trough will be investigated.

\section{Methodology}

\section{a. Numerical model configuration}

The numerical simulations in this study are performed using the Coupled Ocean-Atmosphere Mesoscale Prediction System (COAMPS) for Tropical Cyclones (COAMPS-TC; Doyle et al. 2012, 2014). COAMPS-TC is a specialized version of COAMPS, the navy's operational mesoscale prediction system. The model uses a terrain-following sigma-height coordinate and the nonhydrostatic compressible equations of motion (Klemp and Wilhelmson 1978). A more thorough description of COAMPS is provided by Hodur (1997) and Chen et al. (2003). In this study, all simulations are run at $5-\mathrm{km}$ horizontal resolution. There are 40 sigma levels in the vertical, with a model top of $29.4 \mathrm{~km}$. Consistent with the innermost 5-km nest of the operational COAMPS-TC from 2014 to 2016, no deep convective scheme is employed. However, a parameterization of shallow convection is used. The planetary boundary layer is parameterized using a 1.5-order turbulence closure (Hodur 1997). The model was run with dissipative heating (Jin et al. 2007) but without radiation. Microphysics is specified via prognostic equations for mixing ratios of cloud droplets, ice particles, rain, snow, graupel, and drizzle following Rutledge and Hobbs (1983) and Lin et al. (1983). While COAMPS has the capability of operating fully coupled with the ocean, the simulations presented here are run in uncoupled mode in order to simplify the interpretation of results. With the 

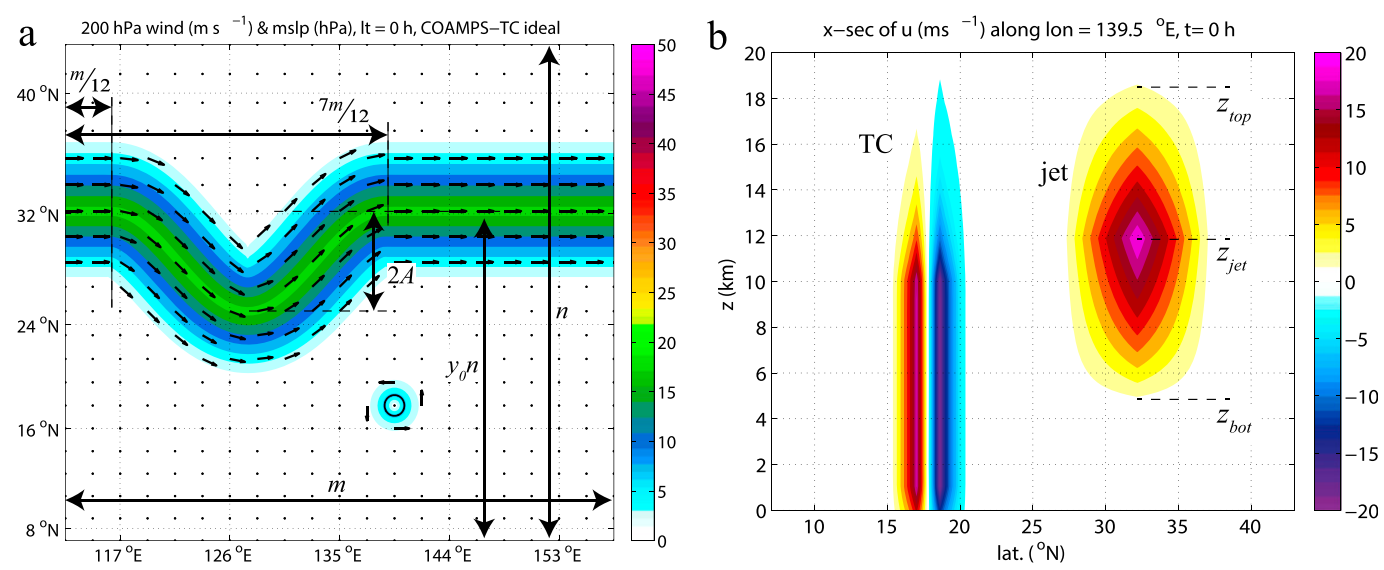

FIG. 1. Schematic depicting construction of the trough and position relative to the TC in (a) the $x-y$ plane on the $200-\mathrm{hPa}$ surface and (b) the $y-z$ plane as a function of latitude $\left({ }^{\circ} \mathrm{N}\right)$ and height $(\mathrm{km})$.

exception of a single simulation run on an $f$ plane, Coriolis is prescribed on a $\beta$ plane.

We initialize the environment using the Jordan (1958) tropical sounding. The surface is initialized without terrain with a uniform SST of $30^{\circ} \mathrm{C}$ to facilitate rapid vortex spinup. The TC is initialized as a modified Rankine vortex, with tangential wind $v_{t}$ specified as a function of radius $r$ given by

$$
v_{t}=\left\{\begin{array}{cl}
v_{\max }\left(\frac{r}{r_{\text {max }}}\right), & r \leq r_{\text {max }} \\
v_{\text {max }}\left(\frac{r_{\text {max }}}{r}\right)^{\alpha}, & r_{\text {max }}<r \leq r_{\text {cut }} \\
0, & r>r_{\text {cut }}
\end{array},\right.
$$

with $\alpha=0.6$, an initial radius of maximum wind $r_{\max }$ of $90 \mathrm{~km}$, a cutoff radius $r_{\text {cut }}$ of $300 \mathrm{~km}$, and a peak tangential wind $v_{\max }$ of $20 \mathrm{~m} \mathrm{~s}^{-1}$ at $900 \mathrm{hPa}$, which decreases linearly to $0.70 v_{\max }$ or $14 \mathrm{~m} \mathrm{~s}^{-1}$ at the surface. Wind is constant from 900 to $300 \mathrm{hPa}$, decreases exponentially from 300 to $180 \mathrm{hPa}$, and is zero above $180 \mathrm{hPa}$. The mass fields are also modified such that the vortex is initialized in thermal wind balance.

\section{b. Trough structure}

A trough is constructed with a cosine-wave structure embedded in a zonal jet. The meridional position of the jet is specified in Cartesian coordinates via the following equation:

$$
y_{\text {jet }}=\left\{\begin{array}{cl}
y_{0} n, & x<\frac{m}{12} \text { or } x>\frac{7 m}{12} \\
A \cos \left(\frac{4 \pi x}{m}+\frac{5 \pi}{3}\right)+y_{0} n-A, & \frac{m}{12} \leq x \leq \frac{7 m}{12}
\end{array},\right.
$$

where $A$ is the trough amplitude, $m$ and $n$ are the zonal and meridional dimensions of the domain, respectively, and $y_{0}$ is the meridional position of the jet (Fig. 1a). In this study, $A$ is set to $400 \mathrm{~km}$ (or $800 \mathrm{~km}$ in $y$ from the zonal jet to the base of the trough), $m$ and $n$ are set to 5005 and $4005 \mathrm{~km}$, respectively, and $y_{0}$ is allowed to vary between 0 and 1 in a set of simulations. The wavelength $\lambda$ is $m / 2$ or $\sim 2500 \mathrm{~km}$. While troughs come in all shapes and sizes, Eq. (4) is designed to generate a "prototypical" trough. The sensitivity of the trough interaction to the amplitude of the trough will be discussed in section 5 , while the sensitivity to the wavelength of the trough will be explored in a future study.
The component of the wind blowing parallel to the line defining the meridional position of the trough [Eq. (4)] is then defined as

$$
v_{\text {jet }}(r)=\left\{\begin{array}{cl}
v_{\text {core }}, & r=0 \\
v_{\text {core }}\left(1-\frac{r}{r_{\text {jet }}}\right), & 0<r \leq r_{\text {jet }}, \\
0, & r>r_{\text {jet }}
\end{array}\right.
$$

where $r$ is distance from $v_{\text {jet }}$ normal to the trough, $v_{\text {core }}$ is the maximum wind at the center of the jet, and $r_{\mathrm{jet}}$ is the cutoff radius. In this study, $v_{\text {core }}$ is set to $20 \mathrm{~m} \mathrm{~s}^{-1}$, and 
$r_{\text {jet }}$ is $600 \mathrm{~km}$. A relatively low value for $v_{\text {core }}$ was chosen through trial and error so as to keep the overall location and structure of the trough relatively stable throughout the simulations. Last, the wind is also scaled in the vertical such that the final wind field is given by

$$
v_{\text {jet }}(r, z)=\left\{\begin{array}{cl}
v_{\text {jet }}(r)\left(\frac{z-z_{\text {top }}}{z_{\text {jet }}-z_{\text {top }}}\right), & z_{\text {jet }} \leq z \leq z_{\text {top }} \\
v_{\text {jet }}(r)\left(\frac{z_{\text {bot }}-z}{z_{\text {bot }}-z_{\text {jet }}}\right), & z_{\text {bot }} \leq z<z_{\text {jet }} \\
0, & z>z_{\text {top }} \text { or } z<z_{\text {bot }}
\end{array},\right.
$$

where $z_{\text {jet }}$ is the height at which the jet is strongest in the vertical and $z_{\text {top }}$ and $z_{\text {bot }}$ are the upper and lower cutoff height levels, respectively, at which the magnitude of the wind associated with the trough goes to zero (Fig. 1b). In this study, $z_{\text {jet }}=11885 \mathrm{~m}(\sigma$ level 10$), z_{\text {top }}=19685 \mathrm{~m}$ $(\sigma$ level 4$)$, and $z_{\text {bot }}=4385 \mathrm{~m}(\sigma$ level 20$)$, or approximately 215,60 , and $600 \mathrm{hPa}$, respectively. Note that our jet decays to zero several kilometers above the surface, similar to Rappin et al. (2011). While midlatitude troughs often extend all the way to the surface, it is not uncommon to have tropical upper-tropospheric troughs (TUTTs) interacting with TCs over tropical waters that are confined entirely to the middle to upper levels (Riehl 1948; Sadler 1967, 1976)

In this study, we assume the jet to be in geostrophic balance and write the momentum equations as

$$
\begin{aligned}
& \frac{\partial u}{\partial t} \sim f\left(v-v_{\text {jet }}\right), \\
& \frac{\partial v}{\partial t} \sim-f\left(u-u_{\text {jet }}\right)
\end{aligned}
$$

(e.g., Yamada and Mellor 1975), where the zonal and meridional components of the jet

$$
\left[\begin{array}{l}
u_{\text {jet }} \\
v_{\text {jet }}
\end{array}\right]
$$

are balanced by a background

$$
\left[\begin{array}{c}
-\frac{\partial \Pi}{\partial y} \\
\frac{\partial \Pi}{\partial x}
\end{array}\right]
$$

where $\Pi=\left(p / p_{0}\right)^{R_{d} / c_{p}}$ is the Exner function, $p_{0}$ is the reference pressure, taken to be $1000 \mathrm{hPa}$ here per convention, $R_{d}$ is the gas constant for dry air, and $c_{p}$ is the heat capacity of dry air at constant pressure (Klemp and Wilhelmson 1978). In diagnosing the balance between the pressure gradient force and Coriolis in the trough as well as the outflow region of the TC, geostrophic wind is computed on constant pressure levels as

$$
\begin{aligned}
& u_{g}=-\frac{g}{f} \frac{\partial Z}{\partial y}, \\
& v_{g}=\frac{g}{f} \frac{\partial Z}{\partial x},
\end{aligned}
$$

where $u_{g}$ and $v_{g}$ are the zonal and meridional components of the geostrophic wind respectively, $g$ is the gravitational acceleration, and $Z$ is the height of the pressure surface (Holton 2004). We compute the geostrophic wind, as opposed to the gradient wind, since the scales in the outflow region tend to be significantly larger than the scale of the vortex itself. Computing geostrophic wind also makes it easier to diagnose the interplay between the TC and the trough, since the trough is assumed to be in geostrophic balance. Last, the ageostrophic wind is computed as

$$
\begin{aligned}
& u_{a}=u-u_{g}, \\
& v_{a}=v-v_{g},
\end{aligned}
$$

where $u_{a}$ and $v_{a}$ are the zonal and meridional components of the ageostrophic wind, respectively. Note that geostrophic balance becomes increasingly invalid close to the equator. As such, we do not compute the geostrophic or ageostrophic wind south of $20^{\circ} \mathrm{N}$ in this study.

\section{Outflow on $f$ and $\beta$ planes}

The purpose of this section is to highlight the key differences between simulated TC outflow $u$ on a $\beta$ plane and an $f$ plane and underscore the realism of the structure of the outflow using the $\beta$-plane approximation relative to an $f$ plane. Two simulations are initialized on a $5005 \mathrm{~km} \times 4005 \mathrm{~km}$ domain with the TC initially located in the center of the grid. The effective latitude of the $f$ plane is set to $20^{\circ} \mathrm{N}$. As expected in the absence of background flow, the TC on the $f$ plane moves little throughout the simulation, remains quite symmetric at the lower levels (Fig. 2c), and steadily intensifies (not shown). This behavior is to be expected (Peng et al. 1999; Rappin et al. 2011) and is quite satisfactory for the purposes of most studies, which utilize idealized TC simulations (e.g., Nolan et al. 2007; Van Sang et al. 2008; Stern and Zhang 2013). However, the upper levels reveal an obvious flaw in the $f$-plane assumption in that the TC develops a symmetric anticyclonic 

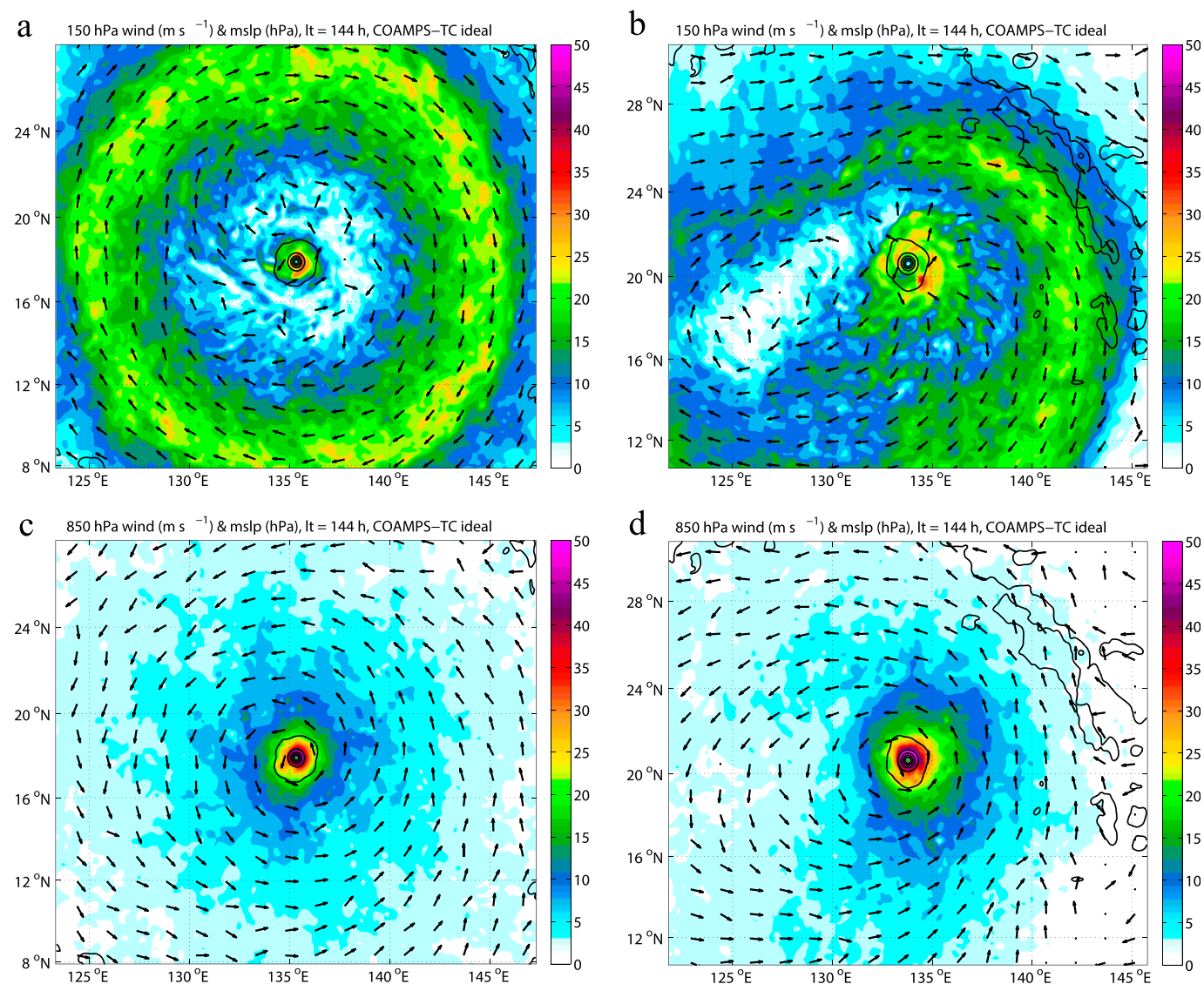

FIG. 2. Wind ( $\mathrm{m} \mathrm{s}^{-1}$; shaded) and mean sea level pressure (MSLP; hPa; contoured in 10-hPa increments at and below $1000 \mathrm{hPa}$ ) at (a),(b) 150 and (c),(d) $850 \mathrm{hPa}$ at $t=144 \mathrm{~h}$ on (a),(c) the $f$ plane and (b),(d) the $\beta$ plane. Latitude-longitude coordinates in the $f$-plane simulation are for display purposes only, since the effective latitude is $20^{\circ} \mathrm{N}$.

tangential wind jet generated by the outflow, centered about the TC at approximately $1000-\mathrm{km}$ radius (Fig. 2a). In the absence of a local minimum in $I$ in the environment, the outflow jet is expected to expand radially outward to the Rossby radius of deformation (Rappin et al. 2011), which is constant with latitude on the $f$ plane given a uniform initial sounding. This structure is quite dissimilar to that observed in real TCs, including what has been observed in studies in which smaller-scale asymmetries are removed by compositing over many cases (e.g., Holland and Merrill 1984; Merrill 1988a,b; Merrill and Velden 1996). Shi et al. (1990), Rappin et al. (2011), and Peng et al. (1999) have all alluded to this deficiency in the $f$-plane assumption.

In contrast with the $f$-plane simulation, the TC on a $\beta$ plane drifts northwestward from its starting location as expected and develops some modest asymmetries in the low-level wind field, particularly at larger radii (Fig. 2d). However, at upper levels, the outflow structure on the $\beta$ plane differs quite notably from the outflow on the $f$ plane. The $\beta$-plane TC features an asymmetric anticyclonic outflow jet that tends to be strongest to the east and south of the TC (Fig. 2b). The fact that the outflow expands to a larger radius equatorward than poleward of the TC on the $\beta$ plane is consistent with the fact that the Rossby deformation radius is inversely proportional to $f$. To further diagnose this outflow asymmetry, 200-100-hPa layer-mean $I$ is computed in a vortexrelative framework in each of these simulations. The fact that there would be stronger outflow directed equatorward than poleward in the $\beta$-plane simulation is consistent with the fact that the outflow tends to occur in regions of lower $I$, which will develop equatorward toward regions of lower $f$ in the absence of environmental flow. There is no net latitudinal (or longitudinal) gradient in $I$ for the $f$-plane simulation (Fig. 3a), implying that there is no favored direction for the outflow to develop. This promotes the development of the anticyclonic jet "ring," driven by the outflow. In contrast, there is an obvious positive poleward gradient in $I$ for the $\beta$-plane 

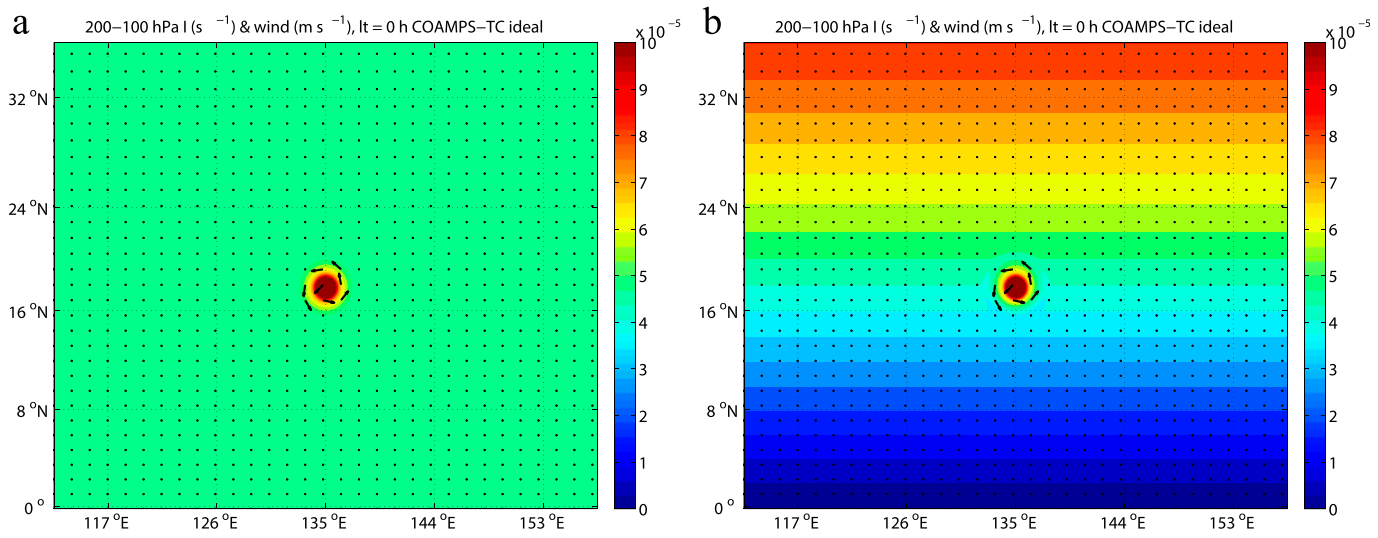

FIG. 3. Layer-mean 200-100-hPa inertial stability $\left(\mathrm{s}^{-1}\right.$; shaded) and wind vectors at $t=0 \mathrm{~h}$ for (a) the $f$-plane and (b) the $\beta$-plane simulations.

simulation (Fig. 3b), which favors greater development of outflow to the south.

These findings suggest that the structure and behavior of the outflow is more realistic when compared to observations (e.g., Merrill 1988a,b) on the $\beta$ plane. Since we seek to explore the effect of trough interaction on outflow structure, amongst other things, the $\beta$ plane will be used in all simulations that follow. It should be noted that while other studies have documented a similar evolution to the outflow on the $\beta$ plane and attributed aspects of the evolution to differences in the gradient of $I$ (e.g., Ge et al. 2008), this discussion serves as a basis for comparison in a more complicated environmental configuration.

\section{TCs and outflow in the presence of a trough}

\section{a. Characteristics of the trough interaction}

For this set of experiments, two new simulations, one with a trough and one without, are initialized with a TC displaced southeast of the center of the domain. The configuration for the simulation with the trough is shown in Fig. 1, while the initial location of the TC is identical in the simulation without the trough. In the trough simulation, the TC is initially located $1300 \mathrm{~km}$ or $0.52 \lambda$ east and $900 \mathrm{~km}$ or $2.25 A$ south of the jet maximum in the base of the trough, where $\lambda$ and $A$ are the wavelength and amplitude of the trough, respectively. These distances are chosen to represent a prototypical interaction between a TC and an approaching trough while allowing the TC enough space to intensify prior to interaction. Experiments with different initial configurations appear in section 5. In diagnosing the evolution of the TC and the environment, we will compute time series of minimum central pressure of the cyclone, 200-100-hPa layermean inertial stability in a $100-500-\mathrm{km}$ annulus centered on the TC, 200-100-hPa layer-mean divergence from
0 - to $500-\mathrm{km}$ radius, and $850-200-\mathrm{hPa}$ vertical wind shear from 0 - to $500-\mathrm{km}$ radius. The $200-100-\mathrm{hPa}$ layer is examined for $I$ and divergence since virtually all of the outflow occurs in this layer in these simulations. The innermost $100 \mathrm{~km}$ are removed in computing $I$ as we are interested in the effect of the environment on $I$, not the effect of the strength of the vortex itself.

The evolution of the TC in the trough and no-trough simulations is extremely similar during the first $60 \mathrm{~h}$, with nearly identical central pressures (Fig. 4a). During this period, the TC in the trough simulation is beginning to enter an environment of reduced 200-100-hPa layermean inertial stability, but initially, this difference is small (Fig. 4b). This azimuthally averaged reduction in $I$ can be directly attributed to an approaching region of reduced $I$ slightly downstream of the base of the approaching trough, northwest of the TC (Figs. 5b,d). The TCs in the two simulations have comparable upper-level divergence signatures (Fig. 4c), and both TCs are initially subjected to very weak vertical wind shear (Fig. 4d). Beyond $60 \mathrm{~h}$, both simulations experience a period of more gradual intensification, with the TC in the trough simulation overall $\sim 5 \mathrm{hPa}$ stronger on average than the TC in the no-trough simulation throughout the 84-132-h period, despite being in an environment of slightly stronger 850-200-hPa shear. During this period, the TC in the trough simulation is in a region of markedly reduced inertial stability and is associated with a distinct burst of upper-level divergence around $96 \mathrm{~h}$. This period from approximately $84-120 \mathrm{~h}$, which commences as the TC comes within $0.25 \lambda$ in $x$ and $1.0 A$ in $y$ of the jet maximum in the base of the trough, will be referred to as the "favorable interaction" phase. During this time frame, the TC is advected northeastward by the trough such that the TC remains several hundred kilometers east of the region of lowest $I$ through the 

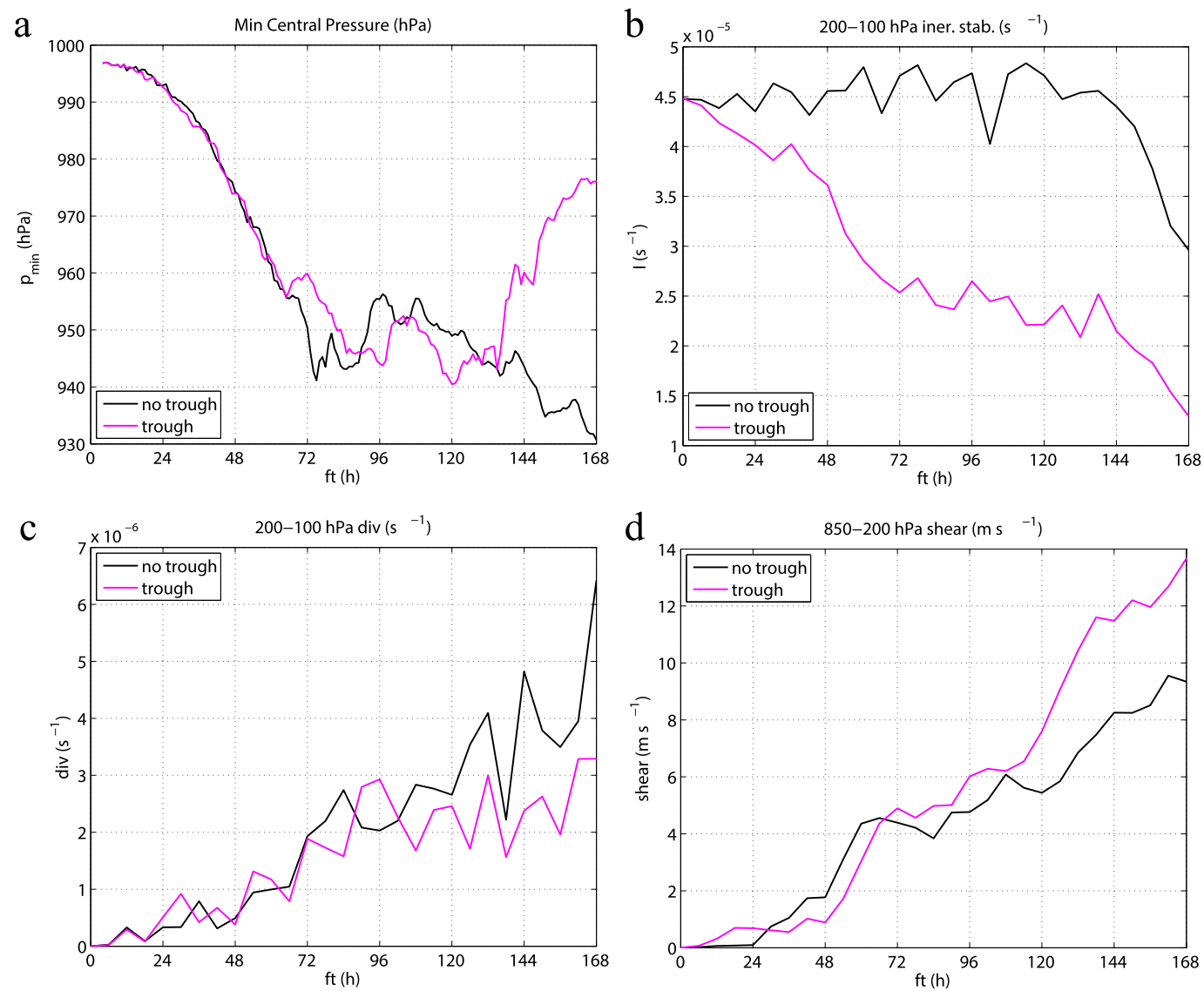

FIG. 4. (a) TC minimum central pressure (hPa), (b) 200-100-hPa layer-mean inertial stability $\left(\mathrm{s}^{-1}\right.$ ) from 100 - to $500-\mathrm{km}$ radius centered on the TC, (c) 200-100-hPa layer-mean divergence $\left(\mathrm{s}^{-1}\right.$ ) from 0 - to $300-\mathrm{km}$ radius, and (d) $850-200-\mathrm{hPa}$ shear $\left(\mathrm{m} \mathrm{s}^{-1}\right)$ from 0 - to $500-\mathrm{km}$ radius, as a function of time (h) for the no-trough (black) and trough (magenta) simulations.

period (Fig. 5f). While the central pressure and divergence time series are noisy during this time frame, we will demonstrate, with the support of sensitivity tests in section 5, that the overall deeper cyclone and the attendant burst of upper-level divergence in the trough simulation are meaningful and not just an artifact of stochastic processes.

Beyond $132 \mathrm{~h}$, the TC in the trough simulation enters a phase that we will refer to as the period of "unfavorable interaction," during which shear continues to steadily increase to unfavorable values of $>10 \mathrm{~m} \mathrm{~s}^{-1}$ (Fig. 4d) and the storm begins to rapidly weaken (Fig. 4a). During this time frame, the TC remains just east of the region of lowest $I$ but is also more directly beneath the stronger jet (Fig. 5h). Relative to the jet maximum in the base of the trough, the TC begins increasing distance in $x$ to $>0.25 \lambda$ while closing the distance in $y$ to zero. In contrast, the TC in the no-trough simulation has moved little, with the minimum $I$ generally occurring equatorward of the cyclone but with a reduction in $I$ with time also occurring poleward of the cyclone as the outflow modifies the environment (Figs. 5a,c,e,g). During the unfavorable interaction phase, the TC in the trough simulation weakens despite the fact that inertial stability continues to drop and upper-level divergence remains comparable to levels that occurred during the favorable interaction phase. In contrast, the TC in the no-trough simulation resumes steadily deepening with upper-level divergence continuing to strengthen during this time frame. Note that shear also increases in the no-trough case, because pronounced upper-level asymmetries develop in the wind field on the $\beta$ plane. However, shear increases more rapidly in the trough simulation.

\section{b. Vertical structure}

Next, we compare the vertical structure of the differences between the trough and no-trough simulations in azimuthal-mean cross sections during the period of favorable interaction. For these analyses, we focus on the northern semicircle of the TC (i.e., $270^{\circ}-90^{\circ}$ clockwise, 
a

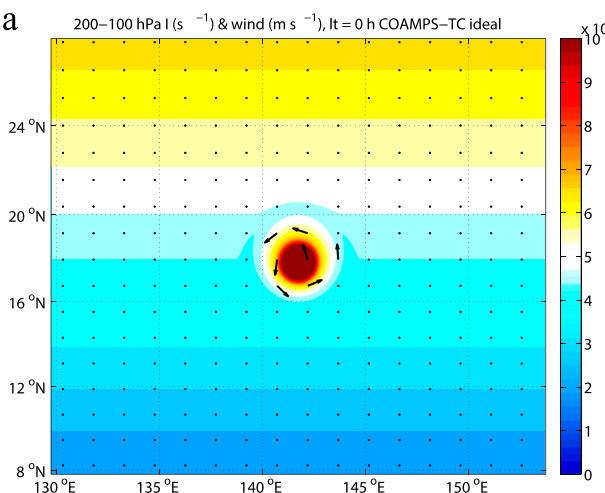

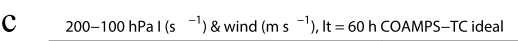

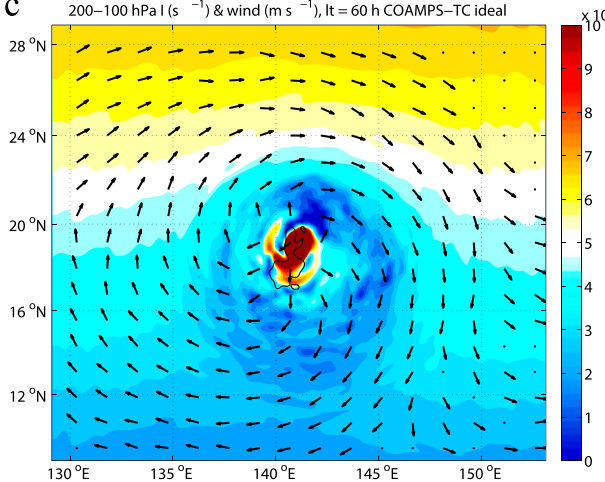

e $\left.200-100 \mathrm{hPal}^{-1} \mathrm{~s}^{-1}\right) \&$ wind $\left(\mathrm{ms}^{-1}\right), \mathrm{It}=120 \mathrm{~h}$ COAMPS-TCideal $\quad \times 10^{-5}$

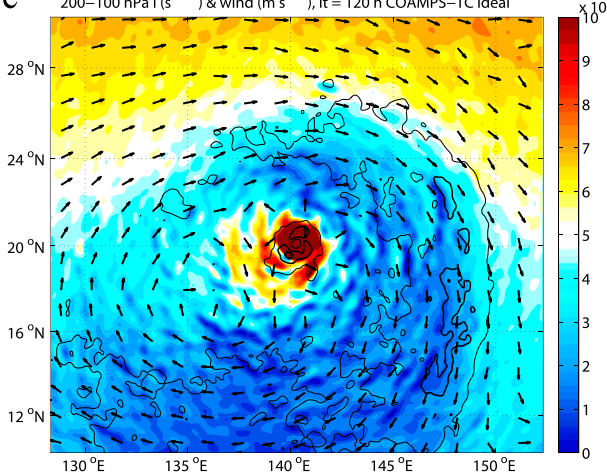

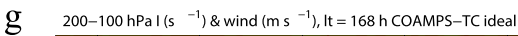

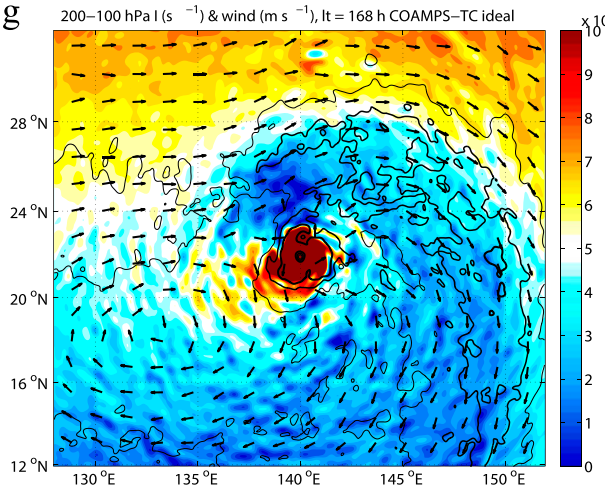

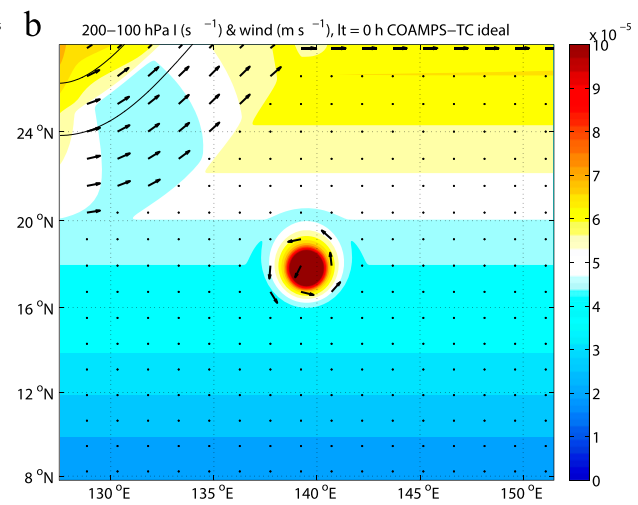
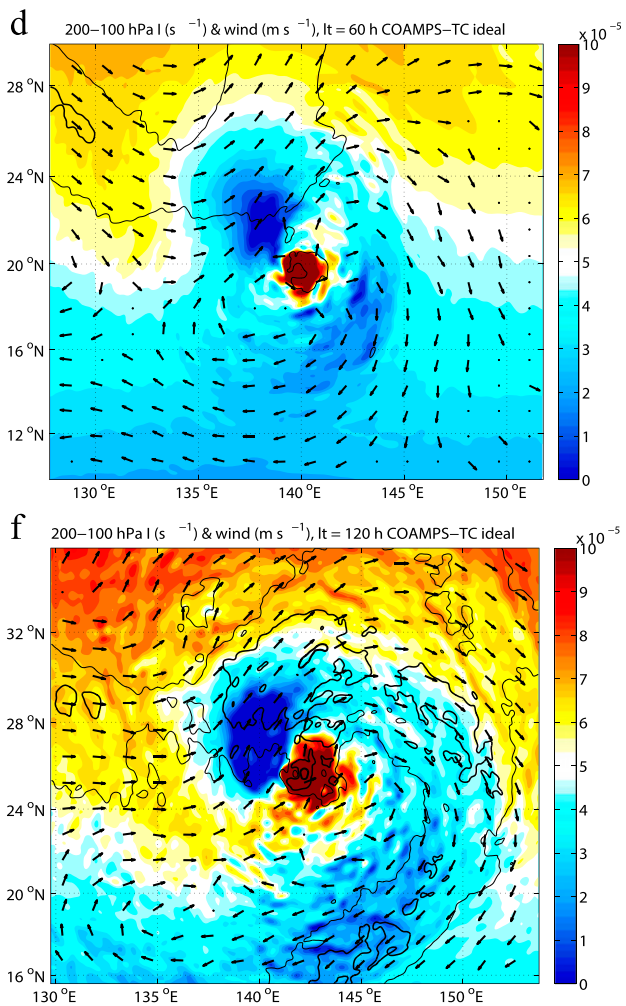

h 200-100 hPal $\left(\mathrm{s}^{-1}\right) \&$ wind $\left(\mathrm{m} \mathrm{s}^{-1}\right), \mathrm{It}=168 \mathrm{~h}$ COAMPS-TCideal

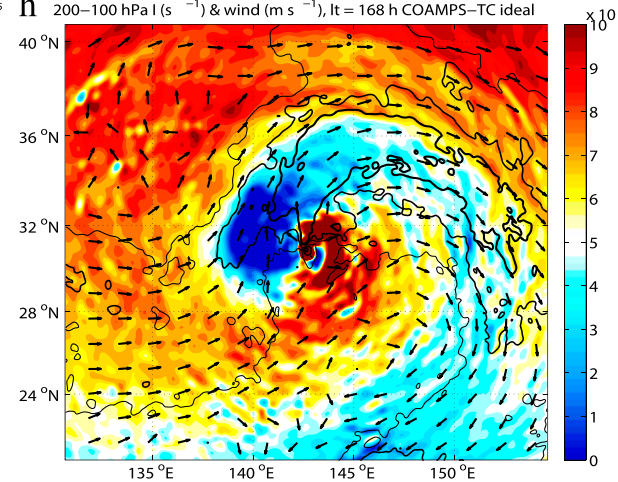

FIG. 5. Layer-mean 200-100-hPa inertial stability ( $\mathrm{s}^{-1}$; shaded), wind $\left(\mathrm{m} \mathrm{s}^{-1}\right.$; contoured every $5 \mathrm{~m} \mathrm{~s}^{-1}$ at and above $10 \mathrm{~m} \mathrm{~s}^{-1}$ ), and wind vectors at $t=$ (a),(b) 0, (c),(d) 60, (e),(f) 120, and (g),(h) $168 \mathrm{~h}$ for (a),(c),(e),(g) the no-trough and (b),(d),(f),(h) the trough simulations. 

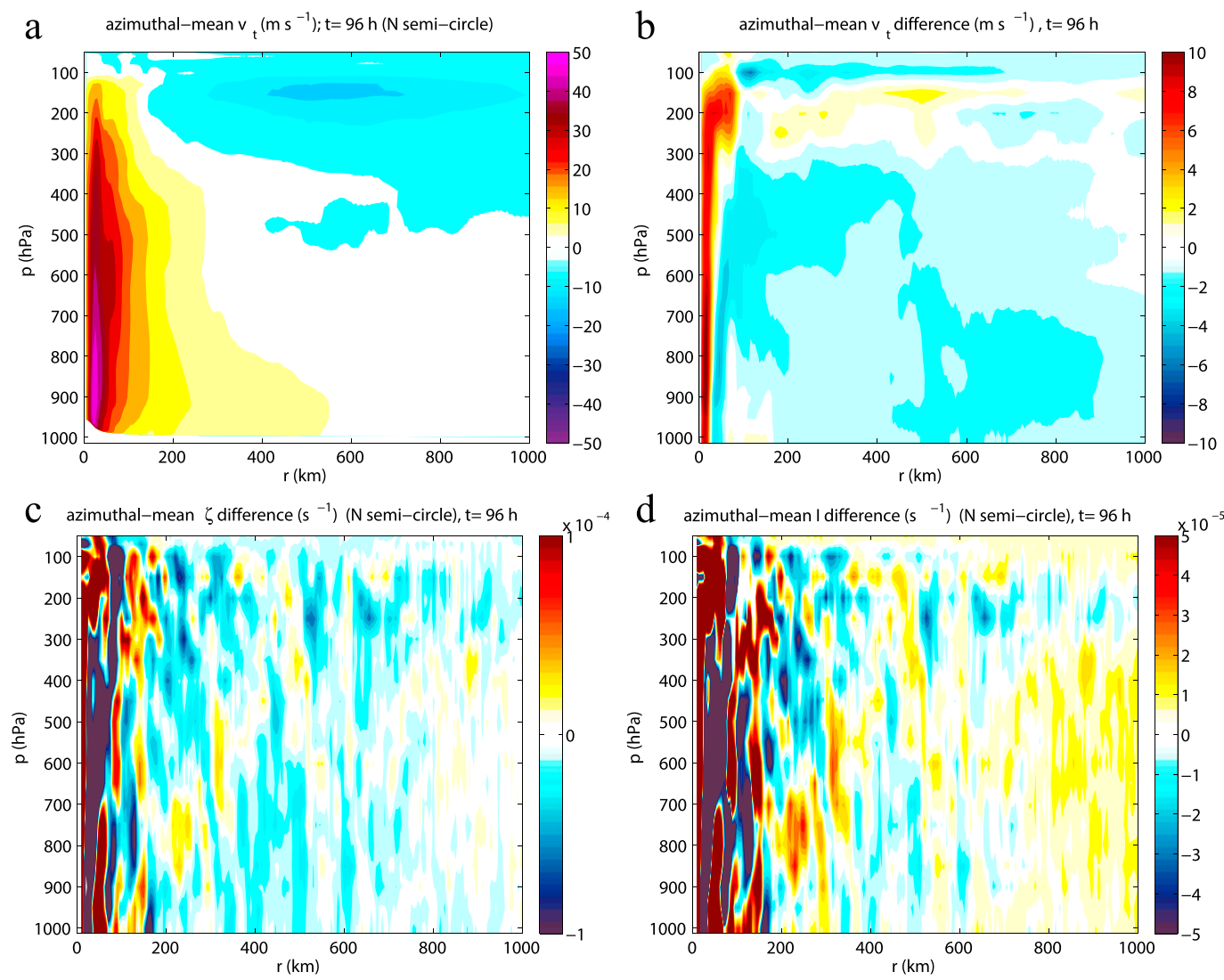

FIG. 6. Azimuthally averaged quantities for the northern semicircle of the TC $\left(270^{\circ}-90^{\circ}\right.$ clockwise $)$ at $t=96 \mathrm{~h}$. Included are (a) $v_{t}\left(\mathrm{~m} \mathrm{~s}^{-1}\right.$, contoured every $\left.5 \mathrm{~m} \mathrm{~s}^{-1}\right)$ for the no-trough simulation, (b) $v_{t}$ difference $\left(\mathrm{m} \mathrm{s}^{-1}\right)$, (c) $\zeta$ difference $\left(\mathrm{s}^{-1}\right)$, and $(\mathrm{d})$ inertial stability difference $\left(\mathrm{s}^{-1}\right)$ between the trough and no-trough simulations (trough minus no trough).

meteorological coordinates) at $96 \mathrm{~h}$. First, it is worth understanding the mean structure of $v_{t}$ for the no-trough simulation before examining difference fields. The TC in the no-trough simulation is associated with cyclonic $v_{t}$ out to $\sim 500 \mathrm{~km}$ at low levels and a tight core of stronger cyclonic wind $>20 \mathrm{~ms}^{-1}$ for radii $<100 \mathrm{~km}$ (Fig. 6a). Negative $v_{t}$ associated with the upper-level anticyclone extends in the vertical from 500 to $50 \mathrm{hPa}$, strongest at $150 \mathrm{hPa}$. The most strongly negative $v_{t}$ of $<-15 \mathrm{~ms}^{-1}$ extends radially from 400 to $800 \mathrm{~km}$ and weakens both closer and farther away from the TC.

Difference fields, computed by subtracting stormrelative fields in the no-trough simulation from the trough simulation, indicate more strongly anticyclonic flow in the trough simulation throughout most of the outflow region $>200 \mathrm{~km}$ from the TC (Fig. $6 \mathrm{~b}$ ). This is intuitive, as enhanced westerly flow north of a TC will be anticyclonic relative to the TC. Differences in $\zeta$ are much noisier but average slightly negative in the outflow layer (Fig. 6c). Overall, this decrease in $v_{t}$ and $\zeta$ north of the TC result in a net reduction in inertial stability from
300 to $100 \mathrm{hPa}$ in the vertical and from $200-$ to $800-\mathrm{km}$ radius (Fig. 6d). Note that the fact that the TC travels significantly farther north in the trough simulation results in greater $f$, counteracting some of the reduction in $I$ associated with the reduction in $v_{t}$ and $\zeta$. However, the net effect on $I$ is still slightly negative. This means that there is less inertial resistance to radial flow in the outflow region north of the TC in the simulation with the trough.

The effect of introducing an asymmetry in the environmental flow on the TC-relative radial wind $v_{r}$ is pronounced. In the no-trough simulation, the strength of the upper-level positive $v_{r}$ (indicative of outflow) at small radii is comparable in the northern (Fig. 7a) and southern (Fig. 7c) semicircles at $120 \mathrm{~h}$. However, the region of positive $v_{r}$ extends radially outward to much larger radius in the southern semicircles: $>1000$ versus $\sim 500 \mathrm{~km}$. In this simulation, stronger outflow is preferentially directed equatorward toward the region of lowest $f$ and thereby lowest inertial stability (Fig. 5e). In contrast, for the TC in the trough simulation during the 

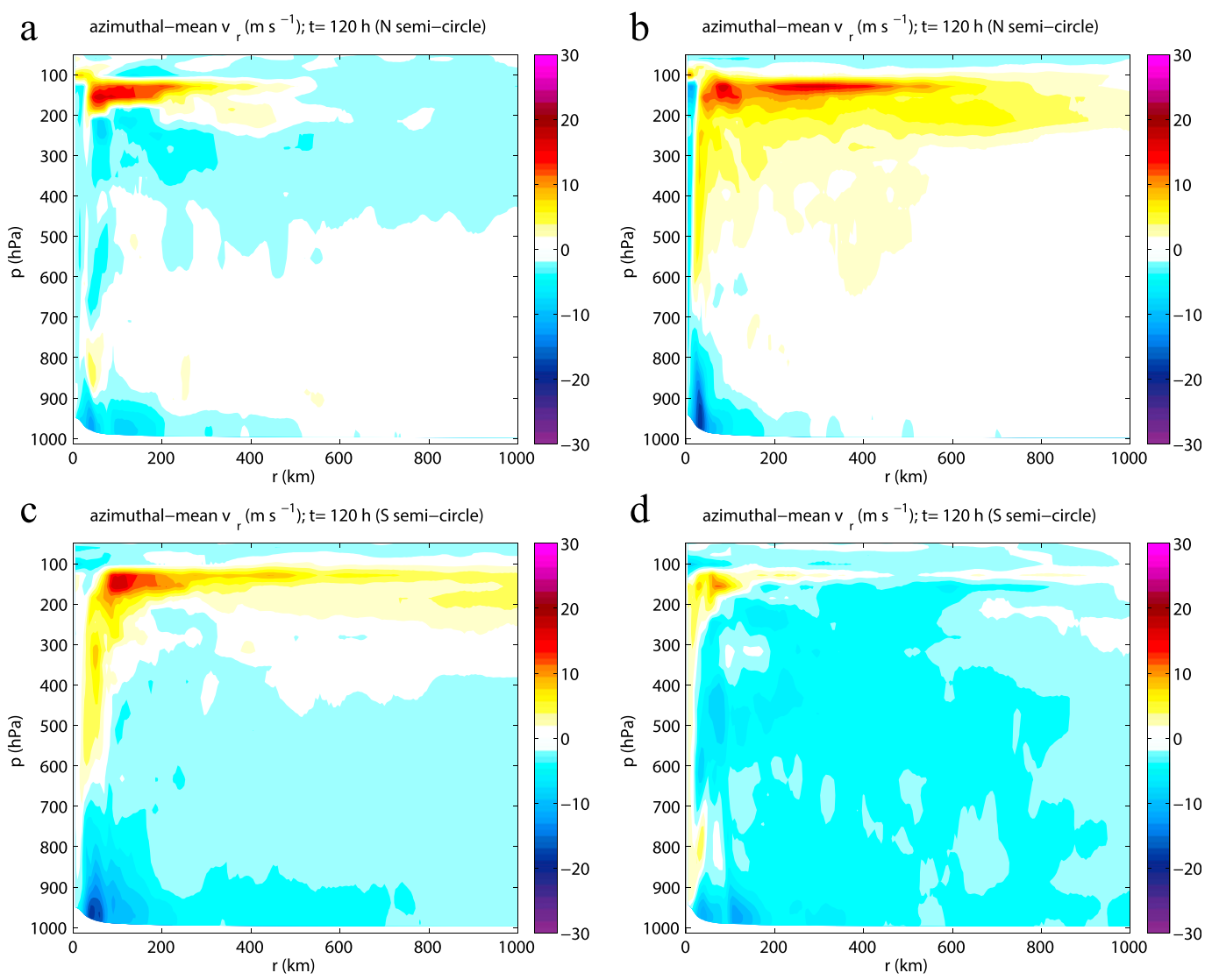

FIG. 7. Azimuthal-mean $v_{r}\left(\mathrm{~m} \mathrm{~s}^{-1}\right)$ for (a),(b) the northern semicircle of the TC $\left(270^{\circ}-90^{\circ}\right.$ clockwise $)$ and (c),(d) the southern semicircle $\left(90^{\circ}-270^{\circ}\right.$ clockwise) at $t=120 \mathrm{~h}$ (note Fig. 6 was at $96 \mathrm{~h}$ ) for (a),(c) the no-trough and (b), (d) the trough simulations.

favorable trough interaction and storm intensification phase, positive $v_{r}$ in the outflow region is both much stronger and extends to much larger radii in the northern semicircle (Fig. 7b) than in the southern (Fig. 7d) semicircle. Once again, the region of strongest outflow occurs in conjunction with the lowest $I$, which in this case is poleward of the TC just ahead of the base of the trough (Fig. 5f).

\section{c. Radial and azimuthal structure}

Earlier results demonstrated a systematic difference between the northward- versus southward-directed outflow between the trough and the no-trough simulations but only at a single forecast time. Radius-time Hovmöller diagrams demonstrate that $200-100-\mathrm{hPa} v_{r}$ is consistently stronger in the southern semicircle (Fig. 8c) than in the northern semicircle (Fig. 8a) throughout the forecast. The opposite relationship is observed in the trough simulation (Figs. 8b,d). Note the strong burst of $v_{r}$ in the northern semicircle from 50 - to $200-\mathrm{km}$ radius that occurs in the trough simulation early in the favorable interaction phase during TC intensification
(Fig. 8b), which corresponds spatially (not shown) and temporally with a local maximum in upper-level divergence (Fig. 4c).

One interesting phenomenon that is noted by Barrett et al. (2016) in their Navy Global Environmental Model (NAVGEM) analyses and is also apparent in our COAMPS-TC simulations is that while the outflow tends to be directed in the approximate direction of lower inertial stability, the strongest radial wind generally occurs about $45^{\circ}-90^{\circ}$ to the right of the actual minimum in $I$. Barrett et al. (2016) attribute this phenomenon to the fact that the outflow is modifying its own environment, primarily via the radial advection of the tangential wind. This acts to reduce (enhance) $v_{t}$ in the equation for $I$ counterclockwise (clockwise) of the region of strongest outflow. The net effect is that the outflow is directed toward a moving target of low $I$ but never reaches it. We would like to refine this result by pointing out that the strongest outflow will occur to the right of the minimum in $I$ even if the outflow were not modifying its own environment. To further investigate this issue, we examine the relationship between the 

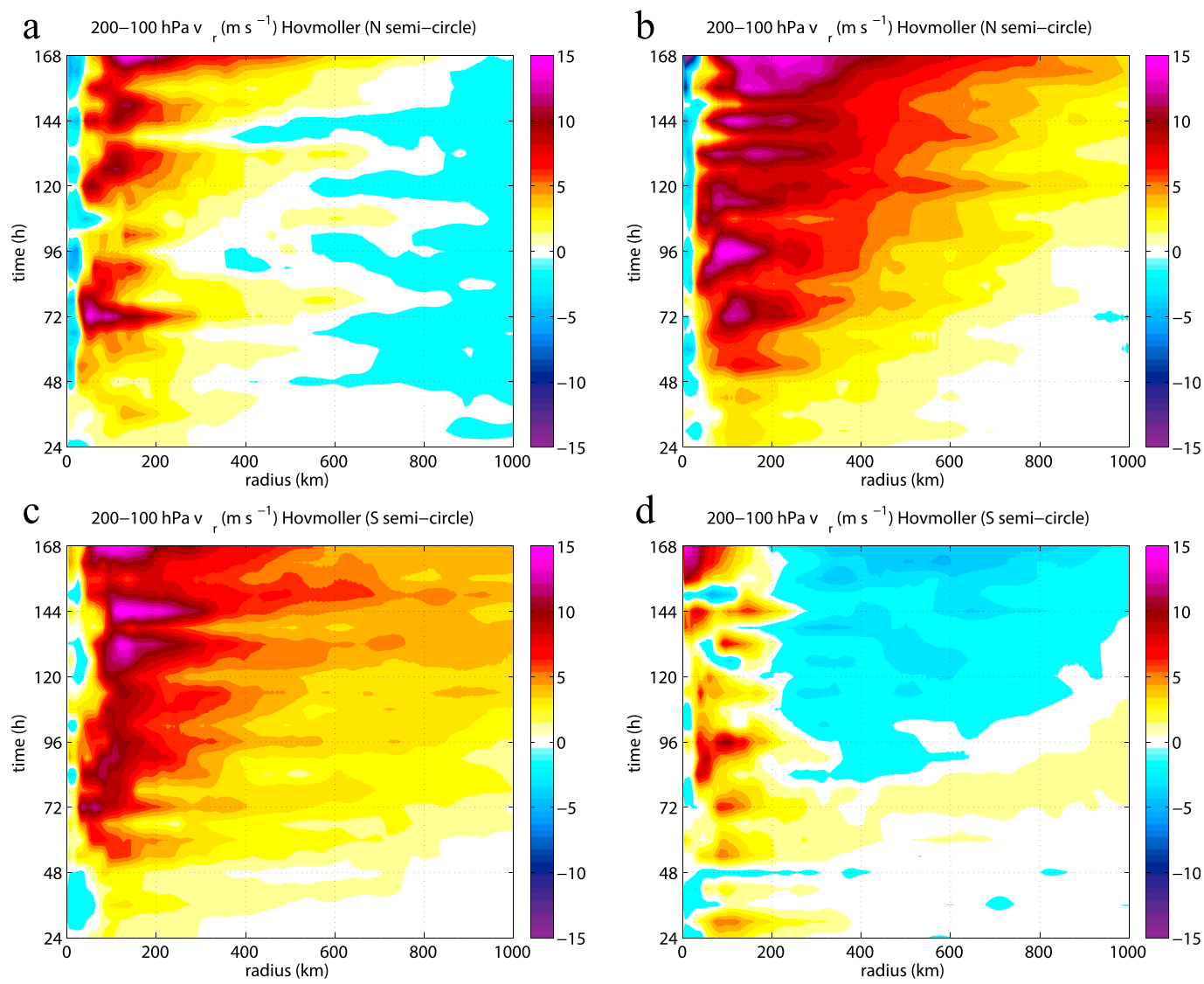

FIG. 8. Azimuthally averaged Hovmöller diagrams of $v_{r}\left(\mathrm{~m} \mathrm{~s}^{-1}\right)$ for the (a),(b) northern semicircle $\left(270^{\circ}-90^{\circ}\right.$ clockwise) and the (c), (d) southern semicircle $\left(90^{\circ}-270^{\circ}\right.$ clockwise) as a function of radius $(\mathrm{km})$ and time (h) for the (a),(c) no-trough and the (b),(d) trough simulations.

pressure gradient, Coriolis, and the outflow characteristics by computing the geostrophic and ageostrophic components of the wind.

As an example in the trough simulation, the $150-\mathrm{hPa}$ wind at $144 \mathrm{~h}$ reveals a distinct maximum in outflow, locally $>25 \mathrm{~m} \mathrm{~s}^{-1}$, almost due north of the TC (Fig. 9a). This region is approximately $45^{\circ}$ to the right of the region of lowest $I$, which occurs northwest of the TC along the TC-trough interface (Fig. 9b). Geostrophic and ageostrophic wind are then computed from Eqs. (8) and (9). The upstream trough and much of the upper-level anticyclonic jet $>300 \mathrm{~km}$ from the TC are all generally in geostrophic balance (Fig. 9c). However, at small radii $<300 \mathrm{~km}$ from the $\mathrm{TC}$, the radial outflow is predominantly ageostrophic (Fig. 9d). Interestingly, a significant fraction of the ageostrophic flow at small radii $\leq 100 \mathrm{~km}$ is directed to the northwest and thereby is in the direction of minimum $I$. By definition, the pressure gradient is too weak to balance the Coriolis force acting on the ageostrophic wind, resulting in a rightward deflection of the wind in the Northern Hemisphere.
Because of this rightward deflection, the region of strongest ageostrophic wind associated with radial outflow rotates from northwest to north with increasing radius. Finally, at larger radii $>300 \mathrm{~km}$, the pressure gradient comes into balance with the Coriolis force, and the wind is in geostrophic balance in the anticyclonic outflow jet (as evidenced by Fig. 11c). However, by this point, the flow is already $45^{\circ}-90^{\circ}$ to the right of the minimum $I$. Put another way, the radial outflow actually is in the direction of lowest $I$ very close to the TC but deflects to the right because the flow is initially unbalanced. Ultimately, the outflow does become quasi balanced but by this point has already deflected $\sim 45^{\circ}$ to the right of the minimum $I$. Since the geostrophic and ageostrophic components of the flow vary as a function of radius, the effect of this radial variation has been overlooked by most other studies that compute radial averages for both $v_{r}$ and $I$.

The time-evolving structure of the geostrophic and ageostrophic wind is examined as a function of azimuth, relative to the minimum in $I$, to determine whether the 

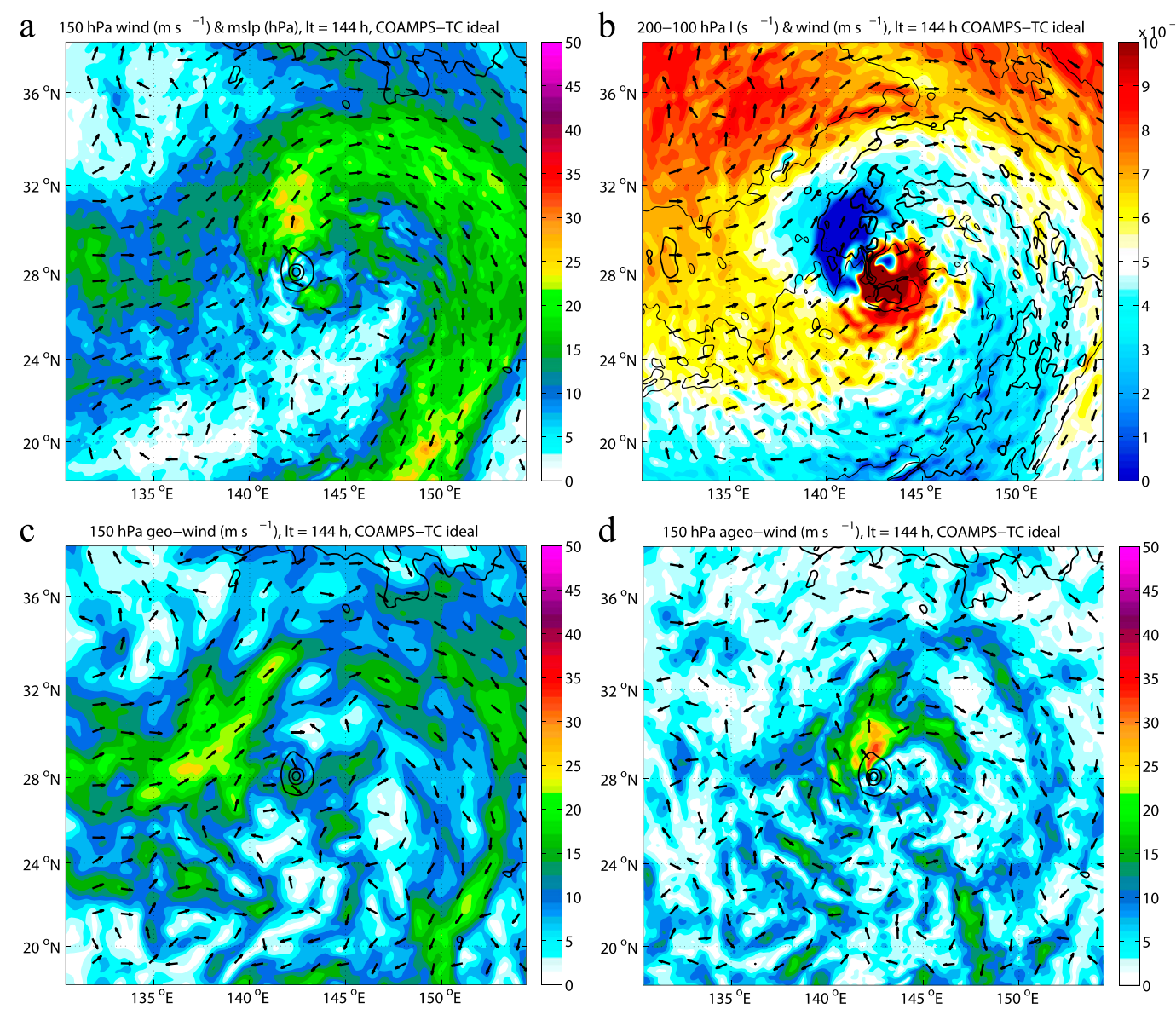

FIG. 9. Zoomed views of (a) wind ( $\mathrm{m} \mathrm{s}^{-1}$; shaded), (c) geostrophic wind ( $\mathrm{m} \mathrm{s}^{-1}$; shaded), and (d) ageostrophic wind ( $\mathrm{m} \mathrm{s}^{-1}$; shaded) with Earth-relative vectors at $150 \mathrm{hPa}$ and MSLP (hPa; contoured every $10 \mathrm{hPa}$ at and below $1000 \mathrm{hPa}$ ) and (b) 200-100-hPa layer-mean inertial stability ( $\mathrm{s}^{-1}$; shaded), wind ( $\mathrm{m} \mathrm{s}^{-1}$; contoured every $5 \mathrm{~m} \mathrm{~s}^{-1}$ at and above $10 \mathrm{~m} \mathrm{~s}^{-1}$ ), and wind vectors. All panels valid at $t=144 \mathrm{~h}$ in the trough simulation.

structure of the outflow at $144 \mathrm{~h}$ is consistent throughout the period of trough interaction. Since it has been established that the radial outflow at small radii is predominantly ageostrophic, and the anticyclonic outflow jet at larger radii is predominantly geostrophic, we focus on the evolution of $150-\mathrm{hPa}$ ageostrophic wind from radius $0-200 \mathrm{~km}$ (Fig. 10a), and the 150-hPa geostrophic wind from radius $500-1000 \mathrm{~km}$ (Fig. 10b). Inertial stability at $150 \mathrm{hPa}$ is computed from 100 - to $500-\mathrm{km}$ radius, with the innermost $100 \mathrm{~km}$ removed so as to prevent the vortex itself from dominating the computation of $I$. The $0-200-\mathrm{km}$ ageostrophic wind at $150 \mathrm{hPa}$ is found to be quite noisy, although the strongest bursts from $100 \mathrm{~h}$ onward (as the TC most closely approaches the base of the trough) most consistently occur from $315^{\circ}$ to $45^{\circ}$ azimuth. This region is generally along or just to the right of the region of lowest $I$. In contrast, the 500-1000-km geostrophic wind develops two distinct maxima at different azimuth beyond $100 \mathrm{~h}$ : one initially around $290^{\circ}$ that rotates counterclockwise to $225^{\circ}$ by $168 \mathrm{~h}$ and another at $340^{\circ}$ that rotates clockwise to $45^{\circ}$ at $168 \mathrm{~h}$. The counterclockwise-rotating geostrophic wind maximum is associated with the upstream jet itself, while the clockwise-rotating maximum is associated with the downstream outflow jet. It is clear in comparing the Hovmöller diagrams of the ageostrophic wind at small radii and the geostrophic wind at larger radii that the geostrophic anticyclonic outflow jet is consistently to the right of the ageostrophic radial outflow from $\sim 100$ to $168 \mathrm{~h}, \sim 30^{\circ}$ to the right at earlier times and $\sim 60^{\circ}$ to the right at later times.

\section{d. Eddy fluxes}

Whether or not EFC during TC-trough interaction promotes storm intensification or structural changes in these simulations is also investigated. A radius-time Hovmöller diagram of 300-100-hPa EFC, centered on the TC in the trough simulation, reveals a steady 

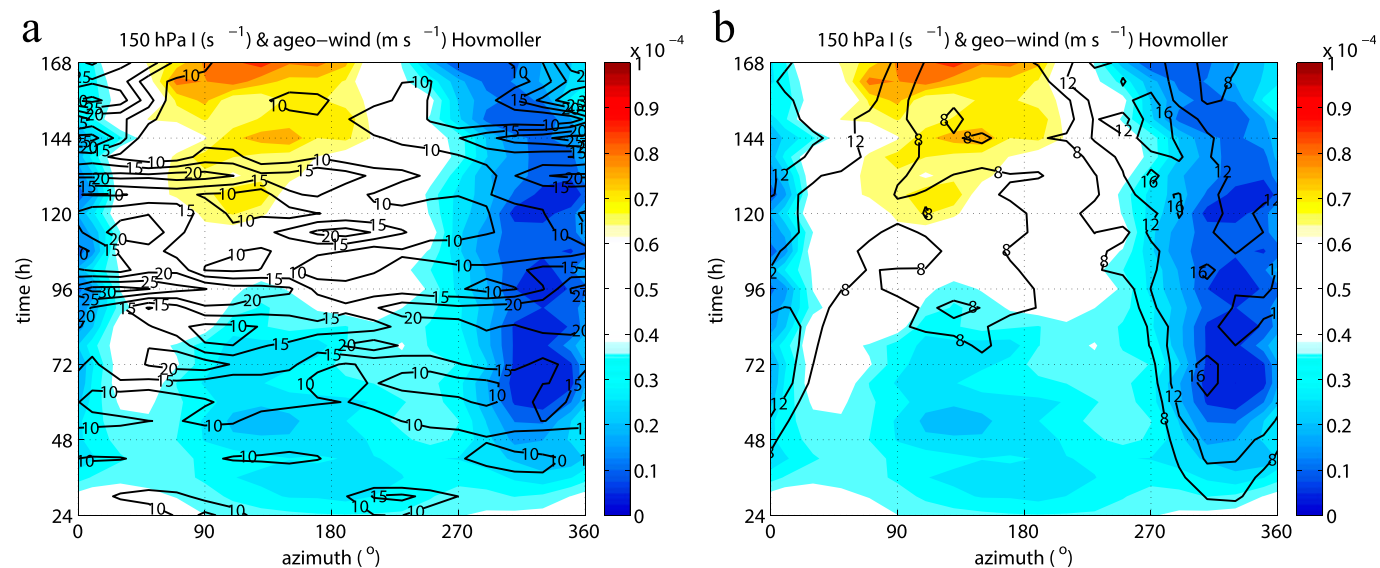

FIG. 10. Hovmöller diagrams of $150-\mathrm{hPa} I\left(\mathrm{~s}^{-1}\right.$; shaded) averaged for $100 \leq r \leq 500 \mathrm{~km}$ as a function of azimuth $\left({ }^{\circ}\right.$; meteorological coordinates) and time (h) for (a) 150-hPa ageostrophic wind ( $\mathrm{m} \mathrm{s}^{-1}$; contoured) for $0 \leq r \leq 200 \mathrm{~km}$ and (b) 150-hPa geostrophic wind ( $\mathrm{m} \mathrm{s}^{-1}$; contoured) for $500 \leq r \leq 1000 \mathrm{~km}$.

increase in eddy fluxes, particularly beyond $72 \mathrm{~h}$ for $r \geq$ $300 \mathrm{~km}$ (Fig. 11a). Earlier studies have used the criteria of $200-\mathrm{hPa}$ EFC $>10 \mathrm{~m} \mathrm{~s}^{-1} \mathrm{day}^{-1}$ from 300 - to $600-\mathrm{km}$ radius to indicate TC-trough interaction (DeMaria et al. 1993; Hanley et al. 2001; Rios-Berrios et al. 2016). In this simulation, this criterion is met in the $300-100-\mathrm{hPa}$ layer (or the 200-hPa level, not shown) at $120 \mathrm{~h}$. In theory, if EFC is directly affecting the outflow, a maximum in $v_{r}$ should appear around this time frame at larger radii and propagate radially inward with time. The balanced
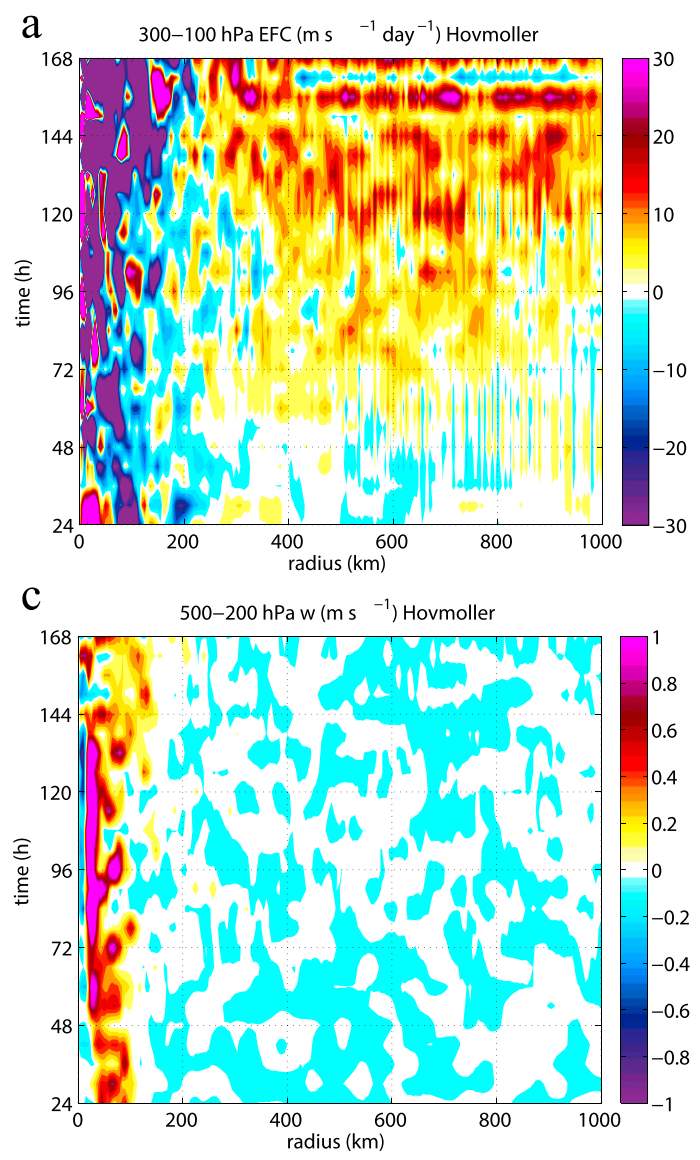

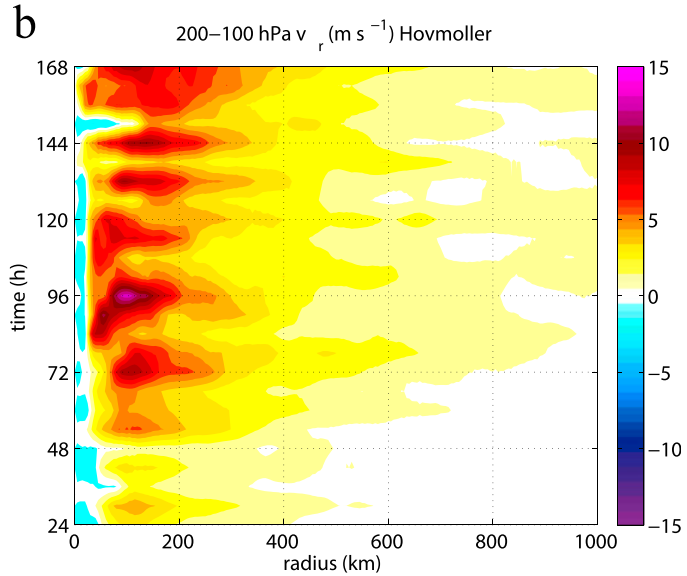

FIG. 11. Hovmöller diagrams of azimuthally averaged (a) 300-100-hPa layer-mean EFC $\left(\mathrm{m} \mathrm{s}^{-1}\right.$ day $\left.^{-1}\right)$, (b) 200-100-hPa layer-mean $v_{r}\left(\mathrm{~m} \mathrm{~s}^{-1}\right)$, and (c) 500200-hPa layer-mean $w\left(\mathrm{~m} \mathrm{~s}^{-1}\right)$ as a function of radius $(\mathrm{km})$ and time $(\mathrm{h})$ for the trough simulation. 
vortex response would produce a local maximum in $w$ there below that also propagates radially inward with time. This inward-propagating region of vertical motion should then trigger a secondary eyewall formation, associated with a temporary weakening of the TC, and followed by a stronger final intensity due to the convergence of angular momentum associated with the eddies (Molinari and Vollaro 1989, 1990).

In this simulation, neither an inward-propagating maximum in azimuthally averaged 200-100-hPa $v_{r}$ (Fig. 11b) or $500-200-\mathrm{hPa} w$ (Fig. 11c) is observed. Instead, the radius of maximum $v_{r}$ is relatively constant but perhaps slightly increases with time from $\sim 100$ to $\sim 200 \mathrm{~km}$ between 96 and $144 \mathrm{~h}$. Vertical motion remains concentrated in the eyewall at low radii $<50 \mathrm{~km}$ through $132 \mathrm{~h}$, with periodic bursts of stronger $w$ from 50- to $100-\mathrm{km}$ radius that do not systematically propagate inward or outward. Vertical motion becomes disorganized beyond $132 \mathrm{~h}$ as the inner eyewall collapses in an environment characterized by stronger vertical wind shear. Therefore, while it is still possible that EFC is affecting the TC structure or intensity, the traditional sequence of events as described by Molinari and Vollaro $(1989,1990)$ by which environmental angular momentum excites secondary eyewall formation and penetrates the core at upper levels does not occur in this simulation. Perhaps the aforementioned evolution only occurs in a subset of TC-trough interaction cases and is more of an anomaly than the norm.

\section{Sensitivity tests}

While we are confident that the reduction in $I$ and increase in vertical wind shear during trough interaction when comparing the trough to the no-trough simulation is both robust and expected, it is more difficult to attribute with certainty the more subtle changes in TC intensity and upper-level divergence directly to trough interaction in the presence of natural nonlinear stochastic processes (e.g., convection). In this section, three sets of experiments are designed to test the sensitivity of the TC to the initial conditions. In the first set of experiments, labeled cases 1-3, the initial latitude of the jet is modified (Fig. 12a). In the second set of experiments, cases $4-6$, the longitude of the TC is varied (Fig. 12b). Last, in cases 7-9, the amplitude of the trough is modified (Fig. 12c). Note that cases 2, 5, and 8 are identical to the trough simulation of section 4 .

First, the effect of varying the initial latitude of the jet is examined. In cases 2 and 3 , the TC begins to accelerate northward as it begins to interact with the slowly eastward-moving trough (Fig. 13a). In case 3, favorable interaction and associated TC intensification occurs from 48 to $72 \mathrm{~h}$, followed by unfavorable interaction and a weakening of the TC from 72 to $144 \mathrm{~h}$ (Fig. 14a). For case 2, favorable interaction occurs from 72 to $120 \mathrm{~h}$, followed by unfavorable interaction from 120 to $168 \mathrm{~h}$. Finally, for case 1, a northward acceleration of the TC never occurs (Fig. 13a), suggesting that the trough is too far north for significant interaction to occur. In this case, the trough misses the TC altogether, and steady intensification occurs through $168 \mathrm{~h}$. The TC in case 3 is stronger than the TC in the other two simulations during favorable trough interaction at $72 \mathrm{~h}$ despite the fact that the other two scenarios are associated with quiescent pretrough environments with lower vertical wind shear (Fig. 14d). From 48 to $72 \mathrm{~h}$, the strongest upper-level divergence (Fig. 14c) and lowest $I$ (Fig. 14b) also occur in case 3, both directly attributable to the trough interaction. Similarly, the TC in case 2 undergoing favorable trough interaction from 72 to $120 \mathrm{~h}$ is stronger than the TC in case 1 in a slightly lower-shear, pretrough environment. At $96 \mathrm{~h}$, upper-level divergence is strongest and $I$ is lowest in case 2. Only after the TCs in cases 2 and 3 weaken beneath the core of the jet does the TC that misses the trough altogether in case 1 become the strongest. The result appears to be robust: the most intense TC at any particular time is the one undergoing favorable trough interaction. The results are also consistent in the sense that TC intensification occurs when the relative distance between the TC and the jet maximum in the base of the trough is between $0.2 \lambda$ and $0.3 \lambda$ in $x$ and $0.8 A$ and $1.2 A$ in $y$ (not shown). During such a configuration, $I$ is generally greatly reduced while upper-level divergence increases, but the increase in vertical wind shear remains small. The time required for favorable interaction to occur in each simulation is proportional to the time it takes for the relative positions of the TC and the trough to achieve these critical distances. Finally, the wind shear rapidly increases and the TC weakens as the separation in $y$ approaches zero as the TC moves under the core of the jet.

Next, the effect of varying the initial longitude of the TC is investigated (Fig. 12b). Similar to what occurred in our last set of sensitivity tests, the TC in cases 4 and 5 begins to accelerate northward as it begins interacting with the trough (Fig. 13b). In the case where the TC is farthest west (case 4), the TC is already nearly south of the trough axis (Fig. 12b). The trough axis remains north of the TC as it slowly drifts eastward, and the TC $\beta$ drifts northward with time. This approach to the trough results in having the $\mathrm{TC}$ be in an environment of increased wind shear (Fig. 15d) with only a modest decrease in $I$ (Fig. 15b). An examination of EFC confirms that minimal interaction with the trough is occurring (not shown) despite the unfavorable increase in shear associated 


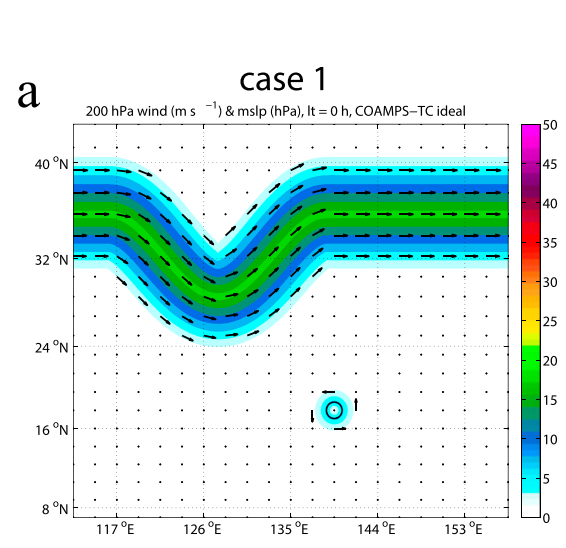

Sensitivity to jet latitude case 2
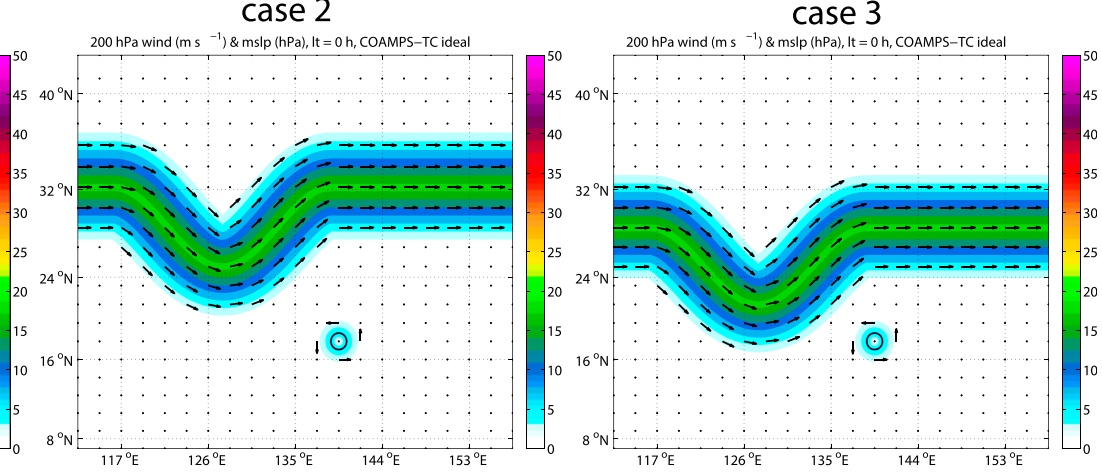

\section{Sensitivity to TC longitude}
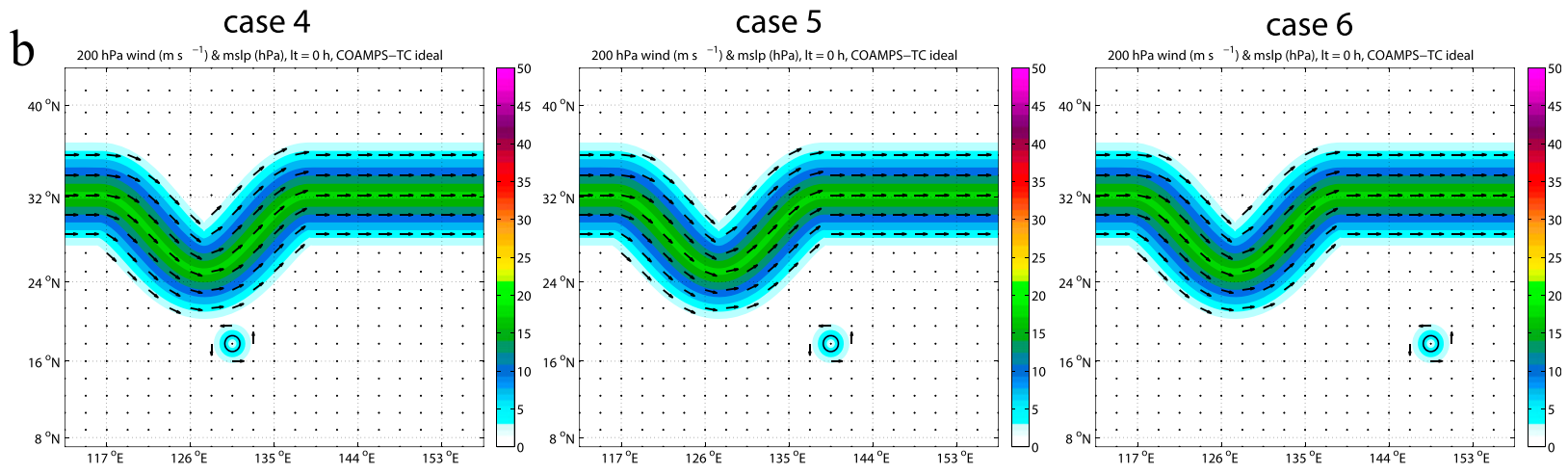

\section{Sensitivity to trough amplitude}

case 7

C

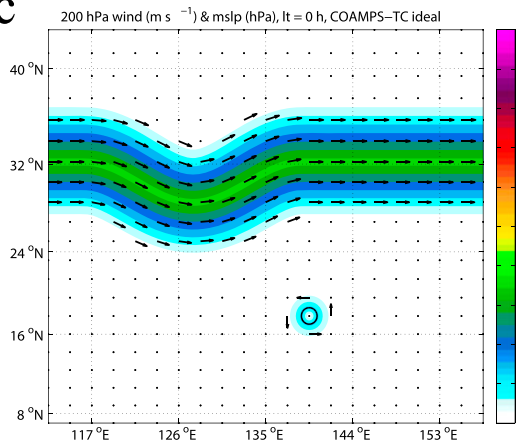

case 8

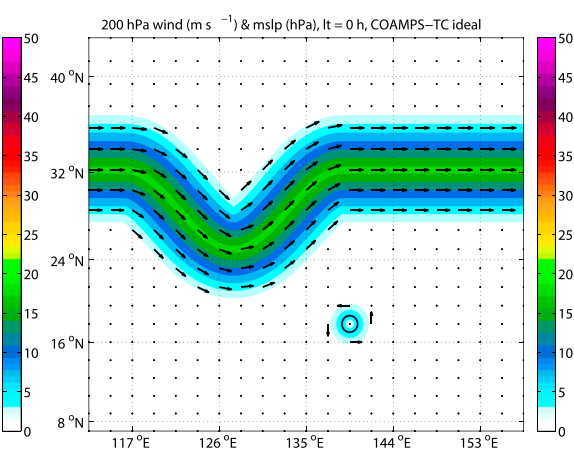

case 9

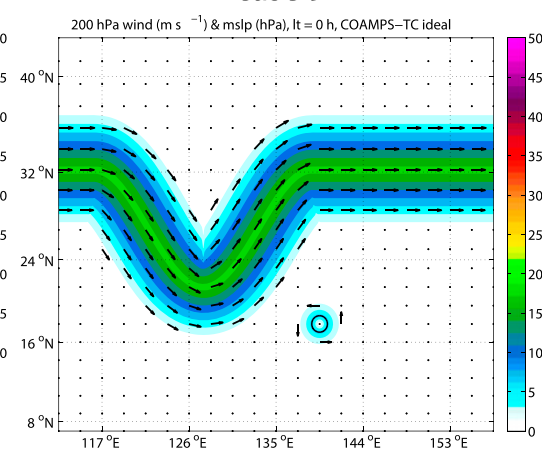

FIG. 12. Initial conditions in various sensitivity experiments: $200-\mathrm{hPa}$ wind $\left(\mathrm{m} \mathrm{s}^{-1}\right)$ and vectors and the $1000-\mathrm{hPa}$ MSLP contour for experiments in which the (a) latitude of the trough is varied, (b) the longitude of the TC is varied, and (c) the amplitude of the trough is varied. Note that the center panel in each row is the control TC-trough simulation from section 4.

with the trough. The net result is minimal intensification throughout the simulation (Fig. 15a). This is a case in which there is no favorable interaction phase, and instead, trough interaction is unambiguously detrimental to TC intensity. The fact that the TC is initially weaker during the period of increasing shear likely contributes to an acceleration of the weakening phase. A number of studies have argued that weaker TCs are more susceptible to moderate vertical wind shear than are stronger
TCs (e.g., Jones 1995, 2000a,b; Reasor et al. 2004; Riemer and Montgomery 2011; Schecter 2015).

Initialized slightly farther east, the $\mathrm{TC}$ in case 5 undergoes a favorable trough interaction from 84 to $120 \mathrm{~h}$ associated with intensification (Fig. 15a) in an environment of low $I$ (Fig. 15b). As previously shown for this case, EFC increases during the period of favorable interaction (Fig. 11a). While there do appear to be stochastic processes during intensification that allow the 

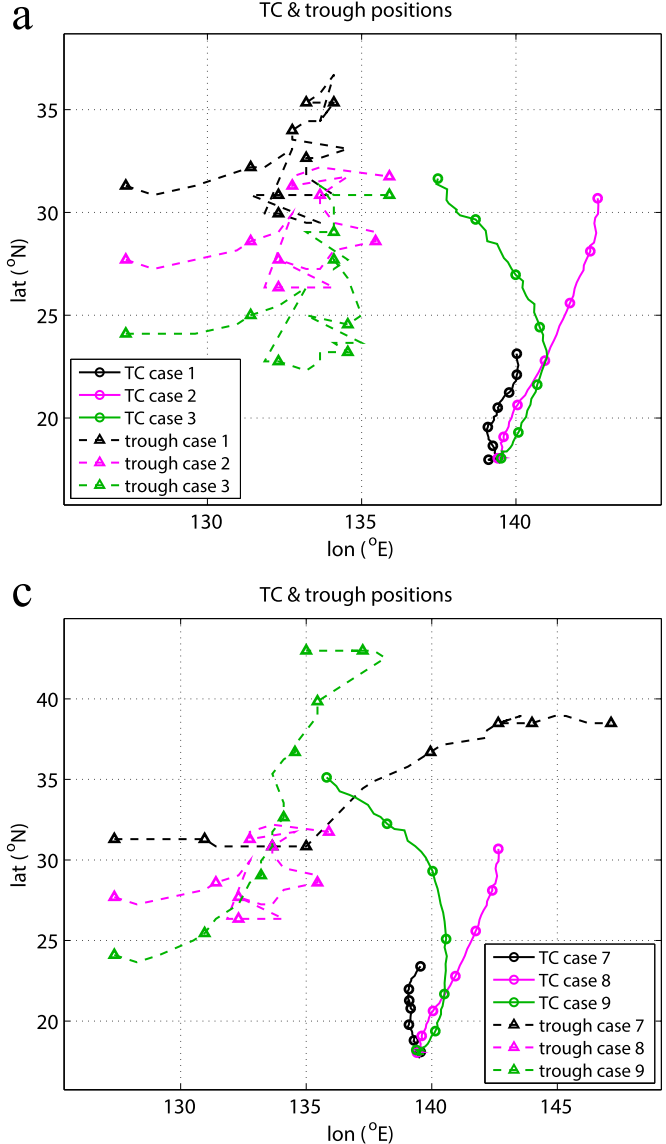

TC in case 6 to briefly ( $<12 \mathrm{~h})$ attain a stronger intensity than the TC in case 5, the TC in case 5 is overall slightly stronger on average throughout the period of favorable interaction. The favorable interaction case is again associated with the strongest upper-level divergence at $96 \mathrm{~h}$ (Fig. 15c) and a deeper cyclone despite there being $4-8 \mathrm{~m} \mathrm{~s}^{-1} 850-200-\mathrm{hPa}$ shear during this period for case 5 versus $2-6 \mathrm{~m} \mathrm{~s}^{-1}$ shear for case 6 .

In the final set of simulations, the sensitivity of the TC-trough interaction to the amplitude of the trough is examined (Fig. 12c). When the trough is amplified (case 9), the effect of the trough on the track of the TC is enhanced (Fig. 13c). Similarly, when the trough is deamplified, the effect of the trough on the TC track is significantly muted. While the more amplified trough certainly reduces the inertial stability (Fig. 16b), the effect of increased vertical wind shear at earlier lead times (Fig. 16d) results in a weakening of the TC (Fig. 16a). Meanwhile, the less amplified trough of case 7 produces a comparable result to the baseline trough of case 8 from 84 to $132 \mathrm{~h}$. The effect on inertial stability in case 7 is subtle: the effect of the trough is just barely able to overcome the competing increase in $f$ as the TC drifts

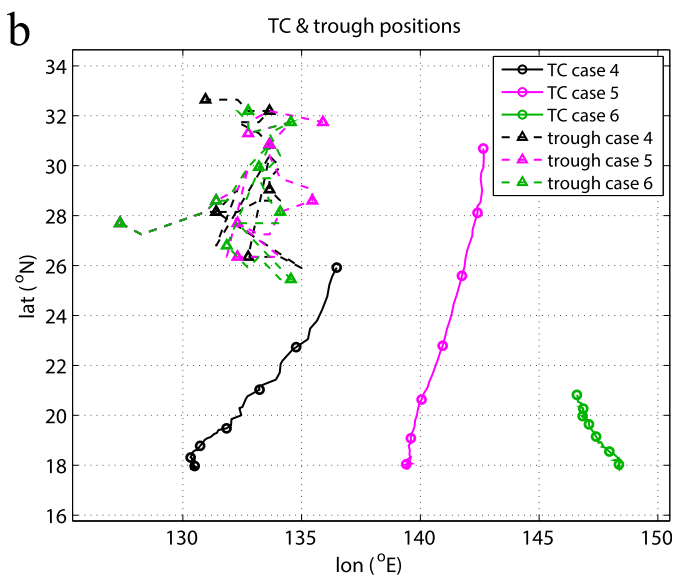

FIG. 13. Tracks through $168 \mathrm{~h}$ of the TC (solid; circles every $24 \mathrm{~h}$ ) and the center of circulation of the trough (dashed; triangles every $24 \mathrm{~h}$ ) in each of the three sets of sensitivity experiments depicted in Fig. 12.

northward, resulting in a very small net reduction in azimuthally averaged $I$ at $96 \mathrm{~h}$. However, a similar increase in upper-level divergence occurs in case 7 as occurs in case 8 (Fig. 16c), and the northward-directed outflow does still strengthen (not shown), suggesting that the deamplified trough is still having an effect on the TC. Further into the simulation, the trough in case 7 continues to progress eastward and passes by the TC entirely. This allows shear to remain low (Fig. 16d) and the TC to continue to intensify (Fig. 16a).

\section{Conclusions}

In this study, we have examined the relationship between environmental inertial stability, TC intensity, and the structure of TC outflow. Simulations were initialized with a $20 \mathrm{~m} \mathrm{~s}^{-1}$ modified Rankine vortex, the Jordan (1958) sounding, and $30^{\circ} \mathrm{C} \mathrm{SSTs}$ that are temporally invariant. We began with the simplest scenario, in which the effect of varying Coriolis on environmental $I$ was investigated. In the absence of background flow on an $f$ plane, environmental $I$ was constant, as expected. As a result, outflow was neither preferentially directed 

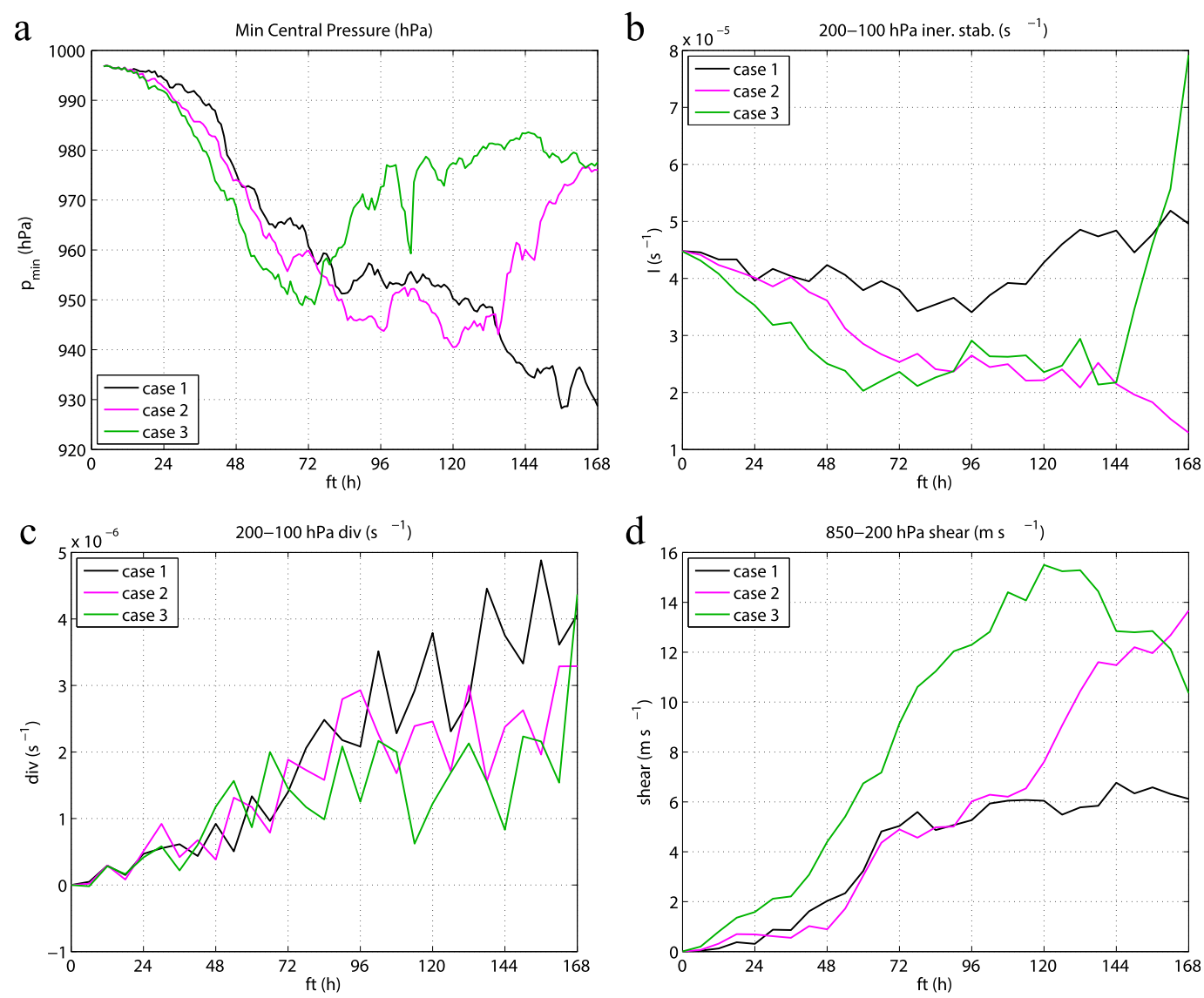

FIG. 14. As in Fig. 4, but for sensitivity experiments in which the latitude of the trough is varied (cases 1-3; Fig. 12a).

poleward nor equatorward relative to the TC. In addition, because the Rossby deformation radius was also constant with latitude, the TC developed a symmetric outflow jet "ring" of radius $\sim 1000 \mathrm{~km}$. In contrast, on a more realistic $\beta$ plane, stronger outflow was directed equatorward in the direction of lower I. Additionally, because the deformation radius decreases with increasing values of $f$, the radius of the upper-level anticyclonic outflow jet also decreased substantially with latitude.

Next, the interaction between a TC and an approaching trough was simulated. The trough is initialized via a cosine function with amplitude $A=400 \mathrm{~km}$ ( or $800 \mathrm{~km}$ from the jet to the base of the trough) and a wavelength $\lambda=2500 \mathrm{~km}$, constructed to represent a prototypical trough. The TC is initially located $1300 \mathrm{~km}$ or $0.52 \lambda$ east and $900 \mathrm{~km}$ or $2.25 A$ south of the jet maximum in the base of the trough. The initial conditions, in particular the relative strength, size, and position of both the TC and the trough, were such that a favorable interaction associated with TC intensification occurred midway through the simulation when the TC was located $\sim 0.25 \lambda$ east and $\sim 1.0 \mathrm{~A}$ south of the jet maximum in the base of the trough. This $24-36-h$ period of intensification was abruptly followed by an unfavorable interaction associated with TC weakening late in the simulation as the meridional separation between the TC and the trough approached zero. Based upon a number of case studies in the literature, this appears to be a plausible scenario in the real atmosphere (Merrill 1988a,b; DeMaria et al. 1993; Hanley et al. 2001).

Relative to the no-trough control simulation, the TC in the trough simulation is in an environment of significantly reduced upper-level $I$ and modestly increased vertical wind shear. Reduced azimuthally averaged $v_{t}$ and $\zeta$ at outflow levels was found to more than compensate for an increase in $f$ for the TC in the trough interaction simulation ( $f$ increases since the TC is advected farther north by the trough), resulting in a net reduction in the azimuthal-mean $I$. As the TC approaches the trough, there is an associated burst of enhanced upper-level divergence at $96 \mathrm{~h}$ and intensification to a comparable, or perhaps slightly stronger, intensity versus the control in a quiescent environment. This period from 84 to $132 \mathrm{~h}$ was referred to as the favorable interaction phase. Beyond $132 \mathrm{~h}$, shear continues to increase as the TC approaches the core of the jet, and the 
a

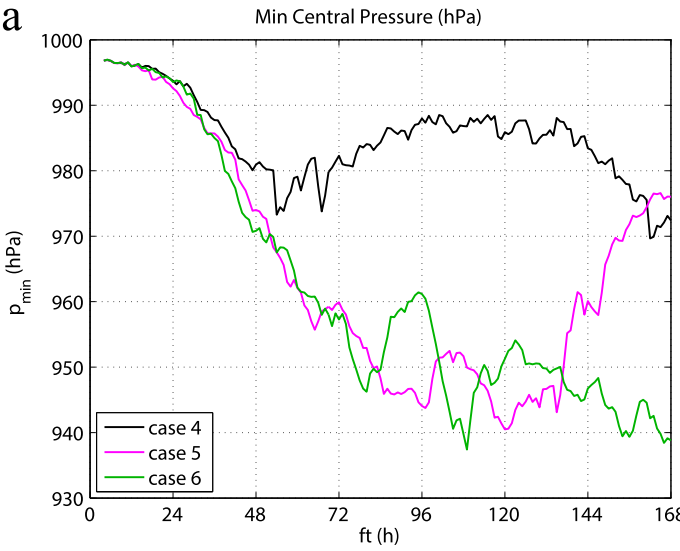

C

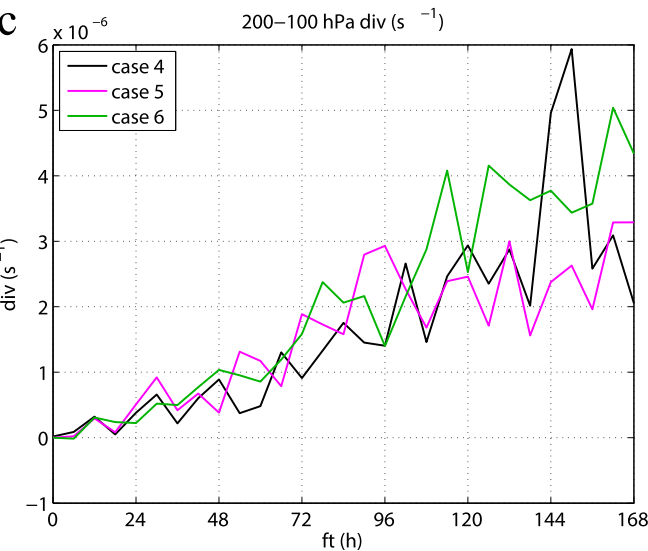

b

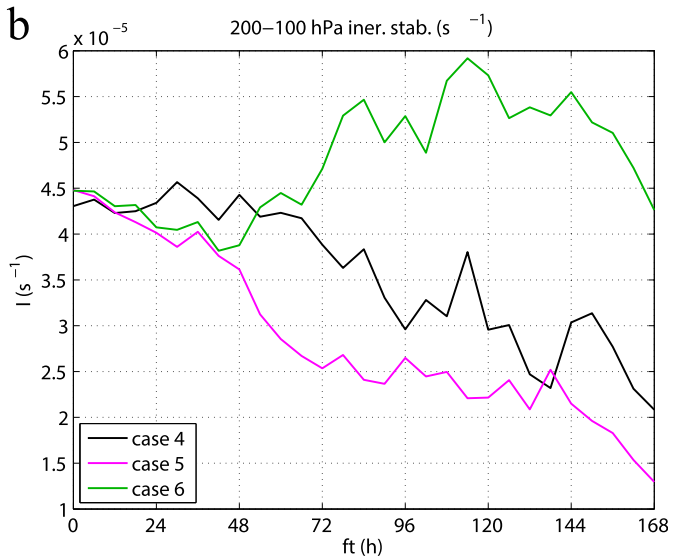

$\mathrm{d}$

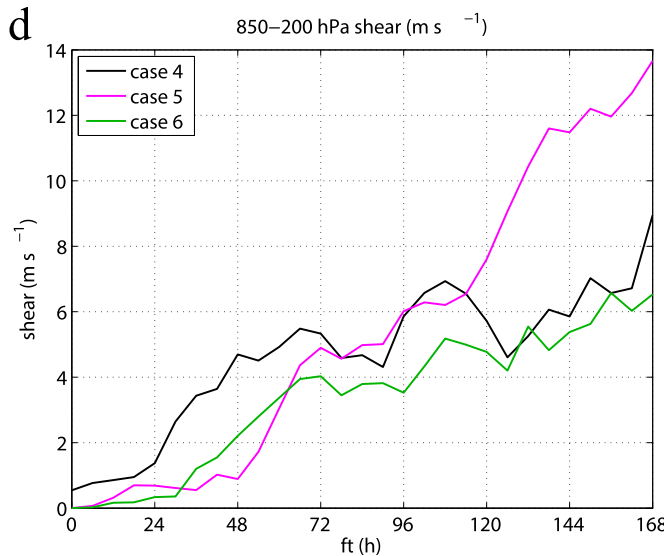

FIG. 15. As in Fig. 4, but for sensitivity experiments in which the longitude of the TC is varied (cases 4-6; Fig. 12b).

storm begins to rapidly weaken. This period was referred to as the unfavorable interaction phase.

During the favorable interaction phase, stronger $v_{r}$ is directed poleward toward the region of lowest $I$ just ahead of the base of the trough. This is in contrast with the control run, in which outflow is directed equatorward toward lower $I$ associated with weaker Coriolis. Overall, the northward-directed outflow in the trough interaction simulation is of comparable magnitude, up to $\sim 15 \mathrm{~m} \mathrm{~s}^{-1}$, to the southward-directed outflow in the control simulation. However, the southward-directed outflow in the control remains stronger, $\geq 5 \mathrm{~m} \mathrm{~s}^{-1}$, out to larger radii than the northward-directed outflow in the trough simulation. This is due to the fact that there is a large, relatively uniform region of low $I$ equatorward of the TC in the control versus a much more concentrated region of low I poleward of the TC in the trough simulation. While the level of strongest outflow of the vertical remains consistent between the two simulations at $\sim 150 \mathrm{hPa}$, the vertical extent of outflow is deeper in the trough simulation, which is likely a consequence of the structure of the trough, which is acting to reduce $I$ over a deeper layer.
An important finding in this study is that the maximum ageostrophic outflow at small radii $\leq 200 \mathrm{~km}$ is actually closer to the direction of the minimum $I$ than is the maximum total wind, which tends to be $45^{\circ}-90^{\circ}$ to the right (in the Northern Hemisphere) of the minimum $I$. However, because there is no pressure gradient to balance the ageostrophic flow, it is deflected to the right. At larger radii $>300 \mathrm{~km}$, much of the outflow and anticyclonic outflow jet are in geostrophic balance, but at these radii, the flow is already away from the $I$ minimum. This effect likely occurs in conjunction with the finding of Barrett et al. (2016) in that the outflow itself modifies the environment, reducing $I$ counterclockwise relative to the region of strongest outflow. Because of both of these reasons, the preferred location of the strongest outflow will tend to be to the right of the region of lowest $I$.

The convergence of 300-100-hPa eddy fluxes was also computed, revealing a meaningful increase in EFC from $300-$ to $600-\mathrm{km}$ radius at and beyond $96 \mathrm{~h}$. While the TC continues to intensify through $132 \mathrm{~h}$, we could not identify a physical mechanism by which the EFC would meaningfully affect the low-level vortex and/or result in vortex spinup. According to Molinari and Vollaro $(1989,1990)$, 

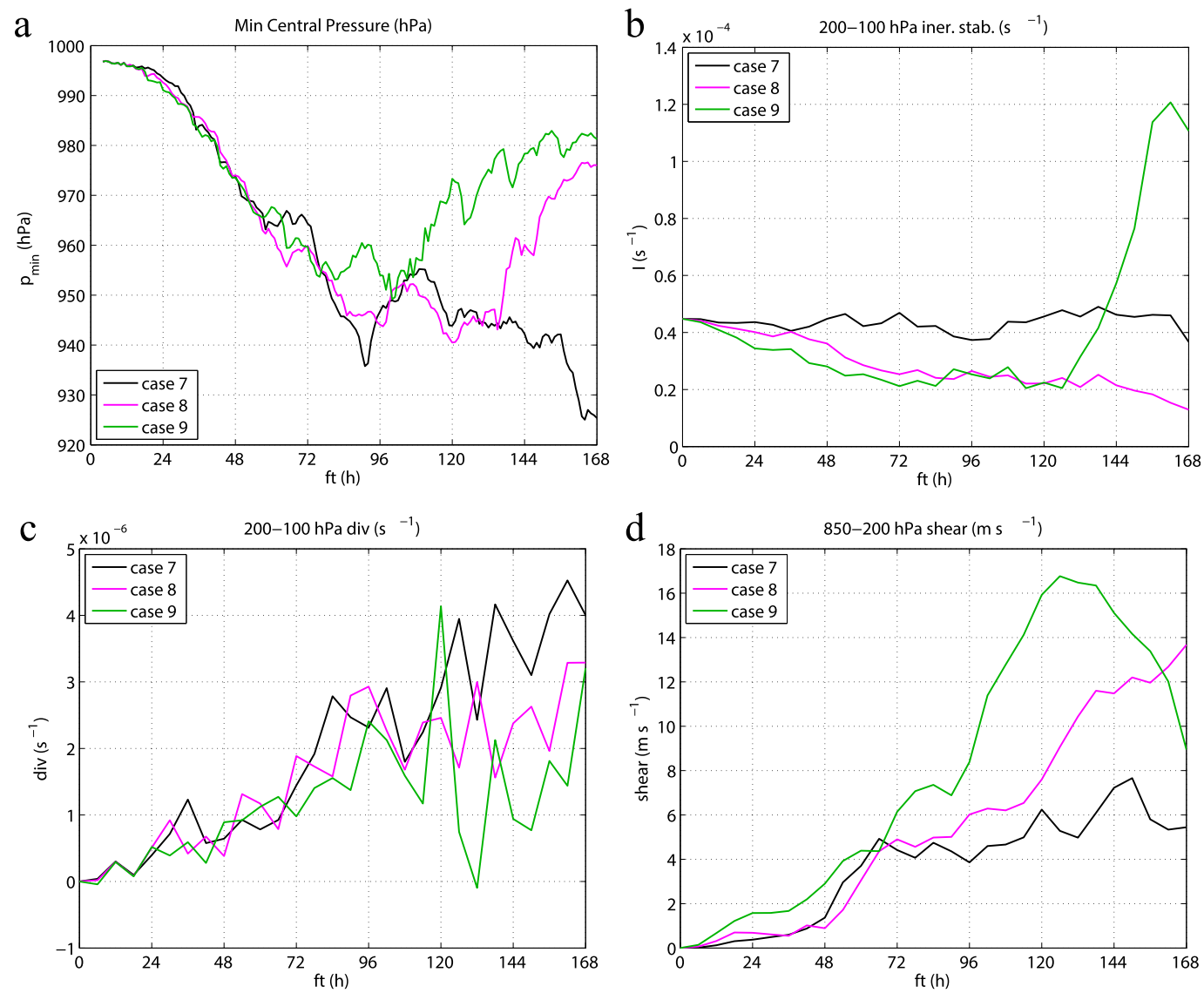

FIG. 16. As in Fig. 4, but for sensitivity experiments in which the amplitude of the trough is varied (cases 7-9;

Fig. 12c).

positive EFC should trigger an inward-propagating circulation associated with enhanced radial wind and vertical motion that initiates a secondary eyewall formation. This has been argued to result in a temporary drop in intensity as the secondary eyewall robs the inner eyewall of momentum and enthalpy flux but a greater final intensity as the secondary eyewall contracts and the angular momentum in the upper-level eddies eventually reaches low radii. However, neither an inwardpropagating region of maximum $v_{r}$ nor $w$ were observed. That said, it is certainly possible that EFC could be a greater contributor to structural or intensity change with a different vortex or trough configuration.

Next, sensitivity tests were performed to tease out differences in intensity fluctuations associated with trough interaction versus more stochastic processes. ${ }^{1}$ For these experiments, the initial latitude of the jet, the initial longitude of the TC, and the amplitude of the

\footnotetext{
${ }^{1}$ A larger "ensemble" of simulations would be necessary to determine a statistically significant difference.
}

trough were varied. Overall, it was found that if the TC is too far south or east of the trough, minimal interaction occurs, and the evolution of the TC is similar to that of the no-trough control. If the TC is initially located too close to the base of the trough, then the storm will be sheared and weaken without ever undergoing a favorable interaction phase. Finally, if the TC is close but not too close to the trough, favorable interaction and intensification of the TC occurs during a 24-48-h time frame. In a set of sensitivity experiments for a real TC case, Leroux et al. (2016) similarly found an optimal TC placement for favorable interaction to occur. In our experiments, TC intensification consistently occurred when the relative separation between the TC and the jet maximum in the base of the trough was $0.2 \lambda-0.3 \lambda$ in $x$ and $0.8 A-1.2 A$ in $y$. These results are generally consistent with past case studies of real TCs. Maximum reduction in inertial stability typically occurs during the favorable interaction stage, as does the strongest upper-level divergence. Thereafter (under our configuration), the TC continues north because of both the $\beta$ effect and southerly flow ahead of the trough and weakens rapidly as the vortex finally succumbs to stronger shear. 
The increase in TC intensity associated with trough interaction, versus a no-trough control or a simulation in which trough interaction has not yet occurred, was often found to be quite subtle, on the order of $5-10 \mathrm{hPa}$ or a few meters per second. However, considering the fact that the TC undergoing trough interaction was always in the environment of greater vertical wind shear, the fact that the interacting TC intensified at a rate comparable to or exceeding that of a $\mathrm{TC}$ in a quiescent environment arguably makes this finding more significant. For several other sensitivity tests, such as when the TC was located directly south of the trough or when the trough amplitude was increased, trough interaction was unfavorable for TC intensification. For both of these cases, the increased vertical wind shear over the TC dominated, and intensification was slowed. The fact that a number of perturbations to our control TC-trough interaction simulation caused the TC to weaken is consistent with DeMaria et al. (1993) and Peirano et al. (2016), both of which found trough interaction to be favorable in some cases but unfavorable in many others. In a sense, the dynamics of the interaction between a trough and an intensifying TC has some overlap with the TT problem. In both cases, trough interaction can be favorable to intensification of the surface vortex but only if the convection associated with the TC is strong enough to overcome negative influences from the trough. While we did not explicitly compute PV in this study, we suspect a similar mechanism to what occurs in the TT studies (Palmén and Newton 1969; Raymond 1992; Posselt and Martin 2004; Davis and Bosart 2003, 2004) is occurring here. We hypothesize that midlevel latent heat release associated with convection acts to erode the upper-level PV anomaly in our "favorable interaction" simulations and hope to show this explicitly in a future study. Presumably if the upper-level PV anomaly is too strong, the TC becomes tilted and asymmetric and weakens. Additionally, convection becomes too weak to build an upper-level anticyclone and fight off the PV anomaly, and the TC weakens further. In future work, a trajectory analysis may be useful in order to diagnose the amount of environmental air that is actually entrained into the core.

Last, in response to a reviewer comment, the sensitivity of the results to the cumulus parameterization was tested. Two simulations with Kain-Fritsch (Kain and Fritsch 1990) cumulus were run: one with the trough and one without the trough. The favorable trough interaction found when no cumulus scheme is used did not occur. Given that only one configuration was rerun with a cumulus parameterization because of computing resources, we hypothesize that a setup that supports a favorable interaction between the TC and the trough with cumulus activated still exists, but activating the cumulus scheme changes the sweet spot. In other words, favorable interaction will likely still occur for a jet of different strength, a trough of different amplitude, or different spacing between the trough and the jet. This result suggests that a future study on the role of convection in TC and trough interactions would help clarify why the results are sensitive to the cumulus parameterization.

The interaction between a TC and a trough is difficult to generalize, as both troughs and TCs come in all shapes and sizes. As noted by Molinari et al. (1995), whether or not TC intensification occurs can be quite sensitive to the strength of the trough. However, the goal of this idealized modeling study has been to explore a small subset of the possible phase space with as few degrees of freedom as possible while still incorporating a fully three-dimensional trough that varies in the $x, y$, and $z$ dimensions. To answer the opening question, we would argue that interactions that affect the outflow do indeed matter for both the overall vortex structure and intensity. In future work, we seek to explore the sensitivity of the TC-trough interaction to the strength of the jet and the tilt of the trough. We also seek to further explore the relationship between outflow and TC intensity change by comparing the results of these simulations to in situ observations from the Tropical Cyclone Intensity (TCI) experiment (Doyle et al. 2017).

Acknowledgments. We thank Daniel Stern of UCAR and three anonymous reviewers for their constructive comments on this work. This research was supported by the NRL Karle's Fellowship program, the Chief of Naval Research through the NRL Base Program, and the Office of Naval Research, PE 0601153N and PE $0602435 \mathrm{~N}$, as part of the Tropical Cyclone Intensity Departmental Research Initiative (TCI-DRI). We acknowledge computational support from a grant of high-performance computing (HPC) time from the Navy Defense Resource Center (DSRC) at Stennis, Mississippi. COAMPS-TC is a registered trademark of the Naval Research Laboratory.

\section{REFERENCES}

Alaka, M. A., 1961: The occurrence of anomalous winds and their significance. Mon. Wea. Rev., 89, 482-494, https://doi.org/ 10.1175/1520-0493(1961)089<0482:TOOAWA >2.0.CO;2.

Barrett, B. S., E. R. Sanabia, S. C. Reynolds, J. K. Stapleton, and A. L. Borrego, 2016: Evolution of the upper tropospheric outflow in Hurricanes Iselle and Julio (2014) in the Navy Global Environmental Model (NAVGEM) analyses and in satellite and dropsonde observations. J. Geophys. Res. Atmos., 121, 13 273-13 286, https://doi.org/10.1002/2016JD025656.

Bentley, A. M., D. Keyser, and L. F. Bosart, 2016: A dynamically based climatology of subtropical cyclones that undergo 
tropical transition in the North Atlantic basin. Mon. Wea. Rev., 144, 2049-2068, https://doi.org/10.1175/MWR-D-15-0251.1.

, L. F. Bosart, and D. Keyser, 2017: Upper-tropospheric precursors to the formation of subtropical cyclones that undergo tropical transition in the North Atlantic basin. Mon. Wea. Rev., 145, 503-520, https://doi.org/10.1175/MWR-D-16-0263.1.

Bosart, L. F., C. S. Velden, W. E. Bracken, J. Molinari, and P. G. Black, 2000: Environmental influences on the rapid intensification of Hurricane Opal (1995) over the Gulf of Mexico. Mon. Wea. Rev., 128, 322-352, https://doi.org/10.1175/ 1520-0493(2000)128<0322:EIOTRI > 2.0.CO;2.

Chen, S., and Coauthors, 2003: COAMPS version 3 model description: General theory and equations. Naval Research Laboratory Tech. Rep. NRL/PU7500-04-448, 141 pp.

Davis, C. A., and L. F. Bosart, 2003: Baroclinically induced tropical cyclogenesis. Mon. Wea. Rev., 131, 2730-2747, https://doi.org/ 10.1175/1520-0493(2003)131<2730:BITC > 2.0.CO;2.

- , and -2004 : The TT problem: Forecasting the tropical transition of cyclones. Bull. Amer. Meteor. Soc., 85, 1657-1662, https://doi.org/10.1175/BAMS-85-11-1657.

DeMaria, M., J.-J. Baik, and J. Kaplan, 1993: Upper-level eddy angular momentum fluxes and tropical cyclone intensity change. J. Atmos. Sci., 50, 1133-1147, https://doi.org/10.1175/ 1520-0469(1993)050<1133:ULEAMF>2.0.CO;2.

Doyle, J. D., and Coauthors, 2012: Real time tropical cyclone prediction using COAMPS-TC. Atmospheric Science $(A S)$ And Ocean Science (OS), C.-C. Wu and J. Gan, Eds., Advances in Geosciences, Vol. 28, World Scientific, 15-28, https://doi.org/ 10.1142/9789814405683_0002.

—_, and Coauthors, 2014: Tropical cyclone prediction using COAMPS-TC. Oceanography, 27 (3), 104-115, https://doi.org/ 10.5670/oceanog.2014.72.

— - and Coauthors, 2017: A view of tropical cyclones from above: The Tropical Cyclone Intensity Experiment. Bull. Amer. Meteor. Soc., 98, 2113-2134, https://doi.org/10.1175/BAMS-D-16-0055.1.

Fischer, M. S., B. H. Tang, and K. L. Corbosiero, 2017: Assessing the influence of upper-tropospheric troughs on tropical cyclone intensification rates after genesis. Mon. Wea. Rev., 145, 1295-1313, https://doi.org/10.1175/MWR-D-16-0275.1.

Galarneau, T. J., R. McTaggart-Cowan, L. F. Bosart, and C. A. Davis, 2015: Development of North Atlantic tropical disturbances near upper-level potential vorticity streamers. J. Atmos. Sci., 72, 572-597, https://doi.org/10.1175/JAS-D-14-0106.1.

Ge, X., T. Li, Y. Wang, and M. S. Peng, 2008: Tropical cyclone energy dispersion in a three-dimensional primitive equation model: Upper-tropospheric influence. J. Atmos. Sci., 65, 2272-2289, https://doi.org/10.1175/2007JAS2431.1.

Guishard, M. P., J. L. Evans, and R. E. Hart, 2009: Atlantic subtropical storms. Part II: Climatology. J. Climate, 22, 3574-3594, https://doi.org/10.1175/2008JCLI2346.1.

Hack, J. J., and W. H. Schubert, 1986: Nonlinear response of atmospheric vortices to heating by organized cumulus convection. J. Atmos. Sci., 43, 1559-1573, https://doi.org/10.1175/ 1520-0469(1986)043<1559:NROAVT>2.0.CO;2.

Hanley, D., J. Molinari, and D. Keyser, 2001: A composite study of the interactions between tropical cyclones and upper-tropospheric troughs. Mon. Wea. Rev., 129, 2570-2584, https://doi.org/10.1175/1520-0493(2001)129<2570: ACSOTI $>2.0 . \mathrm{CO} ; 2$.

Hodur, R. M., 1997: The Naval Research Laboratory's Coupled Ocean/Atmosphere Mesoscale Prediction System (COAMPS). Mon. Wea. Rev., 125, 1414-1430, https://doi.org/10.1175/15200493(1997)125<1414:TNRLSC > 2.0.CO;2.
Holland, G. J., and R. T. Merrill, 1984: On the dynamics of tropical cyclone structural changes. Quart. J. Roy. Meteor. Soc., 110, 723-745, https://doi.org/10.1002/qj.49711046510.

Holton, J. R., 2004: An Introduction to Dynamic Meteorology. 4th ed. Elsevier Academic, 535 pp.

Jin, Y., W. T. Thompson, S. Wang, and C.-S. Liou, 2007: A numerical study of the effect of dissipative heating on tropical cyclone intensity. Wea. Forecasting, 22, 950-966, https:// doi.org/10.1175/WAF1028.1.

Jones, S. C., 1995: The evolution of vortices in vertical shear: Initially barotropic vortices. Quart. J. Roy. Meteor. Soc., 121, 821-851, https://doi.org/10.1002/qj.49712152406.

_ 2000a: The evolution of vortices in vertical shear. II: Largescale asymmetries. Quart. J. Roy. Meteor. Soc., 126, 31373159, https://doi.org/10.1002/qj.49712657008.

- 2000b: The evolution of vortices in vertical shear. III: Baroclinic vortices. Quart. J. Roy. Meteor. Soc., 126, 3161-3185, https://doi.org/10.1002/qj.49712657009.

Jordan, C. L., 1958: Mean soundings for the West Indies area. J. Meteor., 15, 91-97, https://doi.org/10.1175/1520-0469(1958) 015<0091:MSFTWI > 2.0.CO;2.

Kain, J. S., and J. M. Fritsch, 1990: A one-dimensional entraining/ detraining plume model and its application in convective parameterization. J. Atmos. Sci., 47, 2784-2802, https://doi.org/ 10.1175/1520-0469(1990)047<2784:AODEPM>2.0.CO;2.

Kimball, S. K., and J. L. Evans, 2002: Idealized numerical simulations of hurricane-trough interaction. Mon. Wea. Rev., 130, 2210-2227, https://doi.org/10.1175/1520-0493(2002)130<2210: INSOHT $>2.0$. CO;2.

Klemp, J. B., and R. B. Wilhelmson, 1978: The simulation of threedimensional convective storm dynamics. J. Atmos. Sci., $\mathbf{3 5}$, 1070-1096, https://doi.org/10.1175/1520-0469(1978)035<1070: TSOTDC $>2.0 . \mathrm{CO} ; 2$.

Komaromi, W. A., and J. D. Doyle, 2017: Tropical cyclone outflow and warm core structure as revealed by HS3 dropsonde data. Mon. Wea. Rev., 145, 1339-1359, https://doi.org/10.1175/MWRD-16-0172.1.

Leroux, M.-D., M. Plu, D. Barbary, F. Roux, and P. Arbogast, 2013: Dynamical and physical processes leading to tropical cyclone intensification under upper-level trough forcing. J. Atmos. Sci., 70, 2547-2565, https://doi.org/10.1175/JAS-D-12-0293.1.

,-- , and F. Roux, 2016: On the sensitivity of tropical cyclone intensification under upper-level trough forcing. Mon. Wea. Rev., 144, 1179-1202, https://doi.org/10.1175/MWR-D-150224.1.

Lewis, B. M., and D. P. Jorgensen, 1978: Study of the dissipation of Hurricane Gertrude (1974). Mon. Wea. Rev., 106, 1288-1306, https://doi.org/10.1175/1520-0493(1978)106<1288: $\mathrm{SOTDOH}>2.0 . \mathrm{CO} ; 2$.

Lin, Y.-L., R. D. Farley, and H. D. Orville, 1983: Bulk parameterization of the snow field in a cloud model. J. Climate Appl. Meteor., 22, 1065-1092, https://doi.org/10.1175/1520-0450(1983)022<1065: BPOTSF>2.0.CO;2.

Merrill, R. T., 1988a: Environmental influences on hurricane intensification. J. Atmos. Sci., 45, 1678-1687, https://doi.org/ 10.1175/1520-0469(1988)045<1678:EIOHI>2.0.CO;2.

, 1988b: Characteristics of the upper-tropospheric environmental flow around hurricanes. J. Atmos. Sci., 45, 1665-1677, https:// doi.org/10.1175/1520-0469(1988)045<1665:COTUTE > 2.0.CO;2. , and C. S. Velden, 1996: A three-dimensional analysis of the outflow layer of Supertyphoon Flo (1990). Mon. Wea. Rev., 124, 47-63, https://doi.org/10.1175/1520-0493(1996)124<0047: ATDAOT $>2.0 . C O ; 2$. 
Molinari, J., and D. Vollaro, 1989: External influences on hurricane intensity. Part I: Outflow layer eddy angular momentum fluxes. J. Atmos. Sci., 46, 1093-1105, https://doi.org/10.1175/ 1520-0469(1989)046<1093:EIOHIP>2.0.CO;2.

_ , and _ 1990: External influences on hurricane intensity. Part II: Vertical structure and response of the hurricane vortex. J. Atmos. Sci., 47, 1902-1918, https://doi.org/10.1175/ 1520-0469(1990)047<1902:EIOHIP>2.0.CO;2.

— major hurricane. J. Atmos. Sci., 71, 3739-3746, https://doi.org/ 10.1175/JAS-D-14-0117.1.

— S. Skubis, and D. Vollaro, 1995: External influences on hurricane intensity. Part III: Potential vorticity structure. J. Atmos. Sci., 52, 3593-3606, https://doi.org/10.1175/1520-0469(1995) 052<3593:EIOHIP>2.0.CO;2.

Nolan, D. S., E. D. Rappin, and K. A. Emanuel, 2007: Tropical cyclogenesis sensitivity to environmental parameters in environments of radiative-convective equilibrium. Quart. J. Roy. Meteor. Soc., 133, 2085-2107, https://doi.org/10.1002/qj.170.

Nong, S., and K. A. Emanuel, 2003: A numerical study of the genesis of concentric eyewalls in hurricanes. Quart. J. Roy. Meteor. Soc., 129, 3323-3338, https://doi.org/10.1256/qj.01.132.

Palmén, E., and C. W. Newton, 1969: Atmospheric Circulation Systems: Their Structure and Physical Interpretation. Academic Press, 603 pp.

Peirano, C. M., K. L. Corbosiero, and B. H. Tang, 2016: Revisiting trough interactions and tropical cyclone intensity change. Geophys. Res. Lett., 43, 5509-5515, https://doi.org/10.1002/ 2016GL069040.

Peng, M. S., B.-F. Jeng, and R. T. Williams, 1999: A numerical study on tropical cyclone intensification. Part I: Beta effect and mean flow effect. J. Atmos. Sci., 56, 1404-1423, https://doi.org/ 10.1175/1520-0469(1999)056<1404:ANSOTC $>2.0$.CO 2 .

Posselt, D. J., and J. E. Martin, 2004: The effect of latent heat release on the evolution of a warm occluded thermal structure. Mon. Wea. Rev., 132, 578-599, https://doi.org/10.1175/15200493(2004)132<0578:TEOLHR > 2.0.CO;2.

Rappin, E. D., M. C. Morgan, and G. J. Tripoli, 2011: The impact of outflow environment on tropical cyclone intensification and structure. J. Atmos. Sci., 68, 177-194, https://doi.org/10.1175/ 2009JAS2970.1.

Raymond, D. J., 1992: Nonlinear balance and potential vorticity thinking at large Rossby number. Quart. J. Roy. Meteor. Soc., 118, 987-1015, https://doi.org/10.1002/qj.49711850708.

Reasor, P. D., M. T. Montgomery, and L. D. Grasso, 2004: A new look at the problem of tropical cyclones in vertical shear flow: Vortex resiliency. J. Atmos. Sci., 61, 3-22, https://doi.org/ 10.1175/1520-0469(2004)061<0003:ANLATP>2.0.CO;2.

Riehl, H., 1948: On the formation of typhoons. J. Meteor., 5, 247-264, https://doi.org/10.1175/1520-0469(1948)005<0247: OTFOT $>2.0 . \mathrm{CO} ; 2$

_ 1954: Tropical Meteorology. McGraw-Hill, 392 pp.

Riemer, M., and M. T. Montgomery, 2011: Simple kinematic models for the environmental interaction of tropical cyclones in vertical wind shear. Atmos. Chem. Phys., 11, 9395-9414, https://doi.org/10.5194/acp-11-9395-2011.

Rios-Berrios, R., R. D. Torn, and C. A. Davis, 2016: An ensemble approach to investigate tropical cyclone intensification in sheared environments. Part II: Ophelia (2011). J. Atmos. Sci., 73, 1555-1575, https://doi.org/10.1175/JAS-D-15-0245.1.
Rodgers, E. B., S. W. Chang, J. Stout, J. Steranka, and J. J. Shi, 1991: Satellite observations of variations in tropical cyclone convection caused by upper-tropospheric troughs. J. Appl. Meteor., 30, 1163-1184, https://doi.org/10.1175/1520-0450 (1991) $030<1163$ :SOOVIT $>2.0$.CO;2.

Rutledge, S. A., and P. V. Hobbs, 1983: The mesoscale and microscale structure and organization of clouds and precipitation in midlatitude cyclones. VIII: A model for the "seeder-feeder" process in warm-frontal rainbands. J. Atmos. Sci., 40, 1185-1206, https:// doi.org/10.1175/1520-0469(1983)040<1185:TMAMSA > 2.0.CO;2.

Sadler, J. C., 1967: The tropical upper tropospheric trough as a secondary source of typhoons and a primary source of trade wind disturbances. Hawai'i Institute of Geophysics Tech. Rep. 67-12, 44 pp.

_ 1976: A role of the tropical upper tropospheric trough in early season typhoon development. Mon. Wea. Rev., 104, 1266-1278, https://doi.org/10.1175/1520-0493(1976)104<1266: AROTTU $>2.0 . \mathrm{CO} ; 2$.

1978: Mid-season typhoon development and intensity changes and the tropical upper-tropospheric trough. Mon. Wea. Rev., 106, 1137-1152, https://doi.org/10.1175/1520-0493 (1978) $106<1137$ :MSTDAI $>2.0$. CO;2.

Schecter, D. A., 2015: Response of a simulated hurricane to misalignment forcing compared to the predictions of a simple theory. J. Atmos. Sci., 72, 1235-1260, https://doi.org/10.1175/ JAS-D-14-0149.1.

Schubert, W. H., and J. J. Hack, 1982: Inertial stability and tropical cyclone development. J. Atmos. Sci., 39, 1687-1697, https:// doi.org/10.1175/1520-0469(1982)039<1687:ISATCD>2.0.CO;2.

Shapiro, L. J., and H. E. Willoughby, 1982: The response of balanced hurricanes to local sources of heat and momentum. J. Atmos. Sci., 39, 378-394, https://doi.org/10.1175/1520-0469(1982)039<0378: TROBHT $>2.0 . \mathrm{CO} ; 2$.

Shi, J.-J., S. W.-J. Chang, and S. Raman, 1990: A numerical study of the outflow layer of tropical cyclones. Mon. Wea. Rev., 118, 2042-2055, https://doi.org/10.1175/1520-0493(1990)118<2042: ANSOTO $>2.0 . \mathrm{CO} ; 2$.

,$- \ldots$, and - , 1997: Interaction between Hurricane Florence (1988) and an upper-tropospheric westerly trough. J. Atmos. Sci., 54, 1231-1247, https://doi.org/10.1175/15200469(1997)054<1231:IBHFAA > 2.0.CO;2.

Stern, D. P., and F. Zhang, 2013: How does the eye warm? Part I: A potential temperature budget analysis of an idealized tropical cyclone. J. Atmos. Sci., 70, 73-90, https://doi.org/10.1175/JASD-11-0329.1.

Titley, D. W., and R. L. Elsberry, 2000: Large intensity changes in tropical cyclones: A case study of Supertyphoon Flo during TCM-90. Mon. Wea. Rev., 128, 3556-3573, https://doi.org/ 10.1175/1520-0493(2000)128<3556:LICITC $>2.0$.CO;2.

Van Sang, N., R. K. Smith, and M. T. Montgomery, 2008: Tropicalcyclone intensification and predictability in three dimensions. Quart. J. Roy. Meteor. Soc., 134, 563-582, https://doi.org/ 10.1002/qj.235.

Yamada, T., and G. L. Mellor, 1975: A simulation of the Wangara atmospheric boundary layer data. J. Atmos. Sci., 32, 2309-2329, https:// doi.org/10.1175/1520-0469(1975)032<2309:ASOTWA >2.0.CO;2.

$\mathrm{Yu}, \mathrm{H}$., and H. J. Kwon, 2005: Effect of TC-trough interaction on the intensity change of two typhoons. Wea. Forecasting, 20, 199-211, https://doi.org/10.1175/WAF836.1. 